$\widehat{G}_{\text {http://dx.doi.org/10.3765/sp.6.3 }}^{\text {Semantics \& Pragmatics Volume 6, Article 3: 1-57, } 2013}$

\title{
Raising and resolving issues with scalar modifiers*
}

\author{
Elizabeth Coppock \\ University of Gothenburg
}

\author{
Thomas Brochhagen \\ University of Düsseldorf
}

Submitted 2013-02-18 / First decision 2013-04-14 / Revisions received 2013-06-18 / Accepted 2013-07-14 / Final version received 2013-07-21 / Published 2013-08-07

\begin{abstract}
We argue that the superlative modifiers at least and at most quantify over a scale of answers to the current question under discussion (and in this sense, resolve issues), and that they draw attention to the individual possibilities along the scale (and in this sense, raise issues for discussion). The point of departure is a simple analysis on which at least denotes what only presupposes, and at most denotes what only contributes as its ordinary atissue content. This analysis captures the truth conditions, focus-sensitivity, and distribution of superlative modifiers but leaves some pragmatic facts unexplained. We enrich the simple account with unrestricted inquisitive semantics in order to explain the fact that superlative modifiers give rise to ignorance implicatures while comparative modifiers like more and less do not, the fact that superlative modifiers do not give rise to scalar implicatures, and two puzzles concerning the interaction between superlative modifiers and deontic modals. We argue that this proposal provides the most empirically successful published account of superlative modifiers to date.
\end{abstract}

Keywords: numeral, scalar modifiers, QUD, focus, inquisitive semantics, implicature

\section{Introduction}

The reader may find it intuitively obvious that the following two sentences are true under exactly the same circumstances.

* We would like to thank Matthijs Westera, Floris Roelofsen, Kyle Rawlins, Ariel Cohen, Robin Cooper, Hana Filip, Kata Balogh, the audiences at LSA 2013 and the Gothenburg Workshop on Questions and Inquisitive Semantics, three anonymous reviewers, and S\&P editor Louise McNally for discussion and feedback. Research at the University of Gothenburg was funded through the Swedish Research Council grant Semantic Analysis of Interaction and Coordination in Dialogue.

(C)2013 Elizabeth Coppock and Thomas Brochhagen

This is an open-access article distributed under the terms of a Creative Commons NonCommercial License (creativecommons.org/licenses/by-nc/3.o). 
(1) a. John scored at least five goals.

b. John scored more than four goals.

They are both false, for example, if John scored exactly three goals. That intuition can be captured by treating at least $n$ and more than $n$ as generalized quantifiers as follows (Barwise \& Cooper 1981):

(2) $\llbracket$ at least $n \rrbracket=\lambda P . \lambda Q .|P \cap Q| \geq n$

(3) $\llbracket$ more than $n \rrbracket=\lambda P . \lambda Q .|P \cap Q|>n$

However, several researchers have pointed out that things are not quite as simple as they may seem at first glance.

For example, it is often supposed that (4a) semantically entails that Bertha drank three or more beers, and conversationally implicates that she did not drink more, so three is semantically equivalent to at least three. If that is the case, then why doesn't (4b) evoke the same conversational implicature?

(4) a. Bertha drank three beers.

b. Bertha drank at least three beers.

It is usually assumed that three conversationally implicates 'not four' because four is a stronger alternative that the speaker would have chosen had it been appropriate. If that is the case, then, as Krifka (1999) points out, at least three should conversationally implicate 'not at least four' as well by the same logic.

Krifka also points out that at least is focus-sensitive. We offer the following minimal pair to illustrate this:

(5) a. We should at least invite the [postdoc $]_{F}$ to lunch.

b. We should at least invite the postdoc [to lunch $]_{F}$.

(5a) implies that we should invite someone to lunch, while (5b) does not; in (5b) dinner instead would also be fine. On the other hand, (5b) implies that we should invite the postdoc to something, whereas (5a) does not; in (5a) someone more important instead would also be satisfactory. Focus-sensitivity is not expected under the simple generalized quantifier account.

As shown by the previous example, at least and at most do not always modify numerals and can relate to a scale whose elements are not ranked by entailment (Krifka 1999). This point can be made more simply with the following example. 
Raising and resolving issues with scalar modifiers

(6) She is at least an assistant professor.

A higher-ranked alternative in this case would be 'full professor', and being a full professor does not entail being an assistant professor. To put it in Krifka's terms, in general, the scale is ordered by pragmatic strength, which sometimes corresponds to semantic strength (entailment), but not always.

Geurts \& Nouwen (2007) point out a number of differences between superlative modifiers and comparative modifiers. One is that superlative modifiers typically give rise to an ignorance implicature while comparative modifiers do not. Nouwen (2010) gives the following example:

(7) a. \#A hexagon has at least five sides.

b. A hexagon has more than four sides.

Example (7a) is funny because it gives rise to the unlikely implication that the speaker does not know how many sides a hexagon has. (7b) is fine, however, as it does not implicate that.

This contrast manifested itself in an experiment conducted by Geurts, Katsos, et al. (2010), who found that comparative modifiers and superlative modifiers behave differently in inference judgment tasks. Participants in their experiment were given a premise and a conclusion and asked to judge whether the conclusion followed from the premise. Subjects were significantly more likely to judge (8b) as a consequence of (8a) than (8c).

(8) a. Berta had three beers.

b. Bertha had more than 2 beers.

c. Bertha had at least 3 beers.

This can be explained on the grounds that at least gives rise to an ignorance implicature while more than does not. When a statement contains an ignorance implicature, it should be pragmatically odd to express it as a conclusion from a premise that settles the issue.

Geurts \& Nouwen (2007) also point out that superlative and comparative modifiers differ in their distribution:

(9) a. Betty had three martinis \{at most /*fewer than\}.

b. $\quad\{$ At least /*More than\}, Betty had three martinis.

c. Wilma danced with $\{$ at most /*fewer than $\}$ every second man who asked her. 
d. Wilma danced with \{at least /?more than\} Fred and Barney.

While (9) shows that superlative modifiers have a wider distribution than comparative modifiers in some respects, there are also environments where comparatives are more acceptable than superlatives. For example, superlative modifiers seem to resist embedding under negation.

(10) a. John hardly ate \{???at least three / more than two $\}$ apples.

b. This won't take \{???at least 50 / more than 45$\}$ minutes.

(11) a. None of the guests danced with \{???at least three / more than two of the waitresses.

b. Betty didn't have \{???at most three / ?fewer than four $\}$ martinis.

Superlative modifiers also lack certain readings that comparative modifiers can have:

(12) a. You may have at most two beers.

b. You may have fewer than three beers.

While (12b) can be used to grant permission to have fewer than three beers, (12a) can only be used to forbid having more than two. This is Geurts \& Nouwen's (2007) 'missing readings' puzzle.

However, it is important not to restrict the range of readings for superlative modifiers too much. As Büring (2008) points out, the following sentence is ambiguous.

(13) The paper has to be at least 10 pages long.

This sentence has both an 'authoritative reading', on which it informs the interlocutor what the acceptable page lengths are, speaking as the authority on the subject, and a 'speaker insecurity' reading, on which the speaker does not know what the required length of the paper is, but believes it to be over 10 pages.

Let us summarize the main data to be accounted for with a theory of superlative modifiers.

i. Truth conditions: At least three $P$ s are $Q$ s is false whenever fewer than three $P$ s are $Q \mathrm{~s} ;$ At most three $P$ s are $Q \mathrm{~s}$ is false whenever more than three $P$ s are $Q$ s. 
Raising and resolving issues with scalar modifiers

ii. Scalar implicatures: At least three does not give rise to a scalar implicature of no more than three (in contrast with unadorned three).

iii. Focus-sensitivity: Superlative modifiers are focus-sensitive.

iv. Scale types: Superlative modifiers relate to scales that are not necessarily ordered by entailment.

v. Ignorance implicatures: Superlative modifiers give rise to ignorance implicatures and comparative ones do not.

vi. Distribution: Superlative modifiers can modify a range of expressions, not just numerals, and have a wider syntactic distribution than comparative modifiers.

vii. Embedding under negation: Superlative modifiers are less acceptable than comparative modifiers in certain negative environments.

viii. Missing readings: At most lacks a reading under may that fewer than has.

ix. Authoritative readings: At least has both 'speaker insecurity' and 'authoritative' readings under must.

It is quite a challenge to capture all of this in one go, and we will argue in $\$ 4$ that all of the previous proposals (Krifka 1999, Geurts \& Nouwen 2007, Büring 2008, Nouwen 2010, Cummins \& Katsos 2010, Cohen \& Krifka 2011) have missed at least one of these targets.

The proposal we wish to put forth is, we argue, more empirically successful than all of the previous ones (although it is not perfect; we have no explanation for the restricted distribution of superlative modifiers under negation). In §2, we give a preliminary analysis on which at least denotes exactly what only presupposes, and at most denotes exactly what only contributes as its at-issue content, under Coppock \& Beaver's (2011) analysis of only. This analysis (which, for at least, is equivalent to Büring's under certain assumptions) will capture the truth conditions, distribution, flexibility regarding scale types, and focus-sensitivity of superlative modifiers. The story as told in $\$ 2$ fails to account for the facts concerning implicatures, however: sentences with superlative modifiers do not give rise to quantity implicatures (in contrast to corresponding sentences in which the superlative modifiers are omitted), and yet they do give rise to ignorance implicatures (in contrast to corresponding sentences with comparative modifiers). The interactions 
between superlative modifiers and modals also remain mysterious under this view.

In §3, we enrich this account using inquisitive semantics. Essentially, the proposal is that at least $p$ and at most $p$ denote sets of possibilities, where possibilities are sets of possible worlds. At least $p$ denotes the set of answers to the current question under discussion that are ranked as high as or higher than the prejacent in terms of pragmatic strength. At most $p$ denotes the set of possibilities ranked as high as or lower than the prejacent, excluding those possibilities that are ranked higher than the prejacent. This will mean that by uttering a sentence with at least or at most, one is effectively raising an issue for discussion. This is the source of the ignorance implicature. These assumptions can also be used to explain why at least $n$ does not give rise to the same scalar implicature that $n$ gives rise to, and they shed light on the interactions between superlative modifiers and modals.

\section{A QUD-based analysis of superlative modifiers}

As discussed by Coppock \& Beaver (2011, to appear), scalar exclusives like only, just and merely have a presupposition that can be paraphrased with at least, and their ordinary semantic content can be paraphrased with at most. Hence the following inference patterns:

(14) a. This is only/just/merely a down payment.

$\rightarrow$ This is at least a down payment.

$\rightarrow$ This is at most a down payment.

b. This isn't only/just/merely a down payment.

$\rightarrow$ This is at least a down payment.

$\rightarrow$ This is not at most a down payment.

Coppock \& Beaver (2011, to appear) argue that their account of only can explain these patterns. This argument implicitly depends on an analysis of at least and at most that matches their analysis of only. In this section we make that analysis explicit, and enumerate several of its virtues. The analysis in this section will be a starting point for our final analysis, which accounts more successfully for the pragmatic behavior of superlative modifiers. 
Raising and resolving issues with scalar modifiers

\subsection{Information states}

Our analysis of at least (both our preliminary one and the one we will end up with) is based on a pragmatic strength ranking over the answers to the current question under discussion (QUD). Following Roberts (1996/2012) and Beaver \& Clark (2008), and building on Rooth $(1985,1992)$, we assume that the QUD is a set of propositions, and that focus-marking is systematically related to the QUD (more on this in \$2.4). For example, (15a) is felicitous when the QUD is who John invited, while (15b) is not. The QUD for (15b) should be who invited Mary.

(15) a. John invited $[\text { Mary }]_{F}$.

b. $[\mathrm{John}]_{F}$ invited Mary.

We assume that discourse evolves against the background of a discourse context, and that discourse contexts contain a QUD, a ranking over the answers to the QUD, and the common knowledge of the participants. If we have the ranking, we can recover the QUD; it is the set of things that are ranked (since the QUD is a set of propositions, the set of its answers). And from that we can recover the information in the common ground. So we can simply represent contexts (or "information states") as strength orderings over answers to the QUD as in Coppock \& Beaver 2012a.

Foreshadowing the introduction of inquisitive semantics in $\S 3$, we will refer to a set of possible worlds as a 'possibility'. A state $s$, then, is a partially ordered set of possibilities. When we treat a state $s$ as a relation, we write $\geq_{s}$. For example, suppose the question in $s$ is 'Who snores?', and the possible answers are the following, where $w_{10}$ is the world where Ann snores and Bill does not snore, $w_{01}$ is the world where Ann does not snore and Bill snores, and so on:

- $a=\left\{w_{10}, w_{11}\right\}$

'Ann snores'

- $b=\left\{w_{01}, w_{11}\right\}$ 'Bill snores'

- $a \& b=\left\{w_{11}\right\}$

'Ann and Bill snore'

If $a \& b$ is stronger than both $a$ and $b$, and $a$ and $b$ are unranked with respect to each other then we have the following state:

$$
\{\langle a \& b, a\rangle,\langle a \& b, b\rangle,\langle a, a\rangle,\langle b, b\rangle,\langle a \& b, a \& b\rangle\}
$$

In a more visual format: 


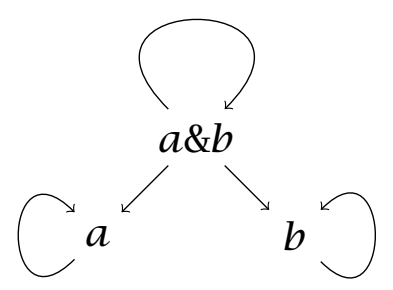

The QUD of $s, \hat{s}$, can be defined as the field of that relation, the set of possibilities that are ordered by the relation (cf. Krifka 1999).

\section{(16) QUD of a state}

If $s$ is a state, then the QUD of $s, \hat{s}=\left\{p \mid \exists p^{\prime}\left[p \geq_{s} p^{\prime}\right.\right.$ or $\left.\left.p^{\prime} \geq_{s} p\right]\right\}$

In our example, the QUD of the state $s$ is:

$$
\hat{s}=\{a, b, a \& b\}
$$

The common ground of $s, s^{*}$, is the union over all possibilities in $\hat{s}$.

\section{(17) Common ground of a state}

If $s$ is a state, then the informational content of $s, s^{*}=\bigcup \hat{s}$

The common ground of a state is a set of indices (possible worlds, for simplicity, here; the framework should ultimately be complicated so that indices are world-assignment pairs). The common ground of the state in our example is $\left\{w_{01}, w_{10}, w_{11}\right\}$. It is in the common ground that presuppositions should be satisfied.

\subsection{Scalar only}

As discussed above in connection with (14), repeated as (18), the presupposition of scalar exclusives like only, just, and merely can be paraphrased with at least, and their at-issue content can be paraphrased with at most (Coppock \& Beaver 2011).

(18) a. This is only/just/merely a down payment.

$\rightarrow$ This is at least a down payment.

$\rightarrow$ This is at most a down payment. 
Raising and resolving issues with scalar modifiers

b. This isn't only/just/merely a down payment.

$\rightarrow$ This is at least a down payment.

$\rightarrow$ This is not at most a down payment.

The at-issue component of only was originally thought to be something that can be paraphrased with nothing/nobody other than instead of at most, and the presupposition was thought to be the prejacent (Horn 1969). As discussed in detail by Beaver \& Clark (2008) and Coppock \& Beaver (2011, to appear), the traditional analysis works for cases like I only invited John, but it doesn't work for cases like (18); (18a) does not imply that this is nothing (relevant) other than a down payment, and (18b) does not imply that this is a down payment (the prejacent). In (18), the salient set of alternatives is not ranked by entailment, but rather by pragmatic strength. When higher-ranked alternatives do not entail lower-ranked alternatives, the inference to the prejacent does not survive negation, as we see in this example.

Beaver \& Clark (2008) analyze only in terms of two meaning components, MIN and MAX, which relate to a pragmatic strength ranking. We adopt the following definitions, based on Coppock \& Beaver 2011.

(19) a. $\quad \operatorname{MIN}_{s}(p)=\lambda w \cdot \exists p^{\prime} \in \hat{s}: p^{\prime}(w) \wedge p^{\prime} \geq_{s} p$

b. $\operatorname{MAX}_{s}(p)=\lambda w . \forall p^{\prime} \in \hat{s}: p^{\prime}(w) \rightarrow p \geq_{s} p^{\prime}$

MIN and MAX are functions from propositions (type $p=\langle s, t\rangle$ ) to propositions. $\operatorname{MIN}_{s}(p)$ says that there is a true answer to the QUD in $s$ that is at least as strong as $p$, and $\operatorname{MAX}_{s}(p)$ says that all answers to the QUD that are stronger than $p$ are false.

Beaver \& Clark's (2008) analysis of only can be stated as follows:

(20) $\llbracket$ only $\rrbracket^{s}=\lambda p .\left\{\begin{array}{l}\operatorname{MAX}_{s}(p), \text { if } s^{*} \vDash \operatorname{MIN}_{s}(p) \\ \text { undefined otherwise. }\end{array}\right.$

The turnstile ' $\models$ ' signifies entailment, and we take this to be defined as subset. As Coppock \& Beaver (2012b) show, this can made more general to account for the fact that only can combine not only with proposition-denoting expressions but also expressions of other types. Coppock and Beaver analyze NP-modifying only as a modifier of generalized quantifiers, and VP-modifying only as a property-modifier. These various uses can be captured under the following schema, where $\tau$ can be any type, and $p=\langle s, t\rangle$ is the type of propositions. 
(21) $\llbracket$ only $\rrbracket^{s}=\lambda \alpha_{\langle\tau, p\rangle} . \lambda \beta_{\tau} .\left\{\begin{array}{l}\operatorname{MAX}_{s}(\alpha(\beta)), \text { if } s^{*} \vDash \operatorname{MIN}_{s}(\alpha(\beta)) \\ \text { undefined otherwise. }\end{array}\right.$

This variant can be obtained using the Geach rule (e.g. Jacobson 1999). The Geach rule converts a function $f$ with type $\langle a, b\rangle$ into a function $f^{\prime}$ with type $\langle\langle c, a\rangle,\langle c, b\rangle\rangle$ of the form $\lambda R . \lambda x . f(R(x))$, where $R$ has type $\langle c, a\rangle$ and $x$ has type $c$. If $a$ and $b$ are $p$, and $c$ is type $\tau$, then we obtain the type of variant given in (21).

\subsection{Lexical entries for superlative modifiers}

As mentioned above, Krifka (1999) pointed out that, just as with only, the focus alternatives used by superlative modifiers are ranked by pragmatic strength, which can coincide with semantic strength (entailment), but need not do so. For example, in (22), the question under discussion is something like 'What position does John hold?'.

(22) John is at least an [assistant professor $]_{F}$.

The answers include, in order of strength, 'John is a grad student', 'John is a postdoc', 'John is an assistant professor', 'John is an associate professor', and 'John is a full professor'. What the sentence means can be expressed as a disjunction over the answers that are at least as strong as 'John is an assistant professor': 'John is an assistant, associate, or full professor'. The answers to the question under discussion in this case are mutually exclusive; one cannot be a full professor and an assistant professor at the same time. So the stronger answers do not entail the weaker answers in this example. We can account for this by assuming that superlative modifiers make use of the same kind of scales that only uses.

Specifically, let us assume that, as Coppock \& Beaver (2011) argue, at least denotes what only presupposes and at most expresses only's ordinary semantic content, so at least denotes MIN and at most denotes MAX. To be more precise, in state $s$, at least $p$ means $\operatorname{MIN}_{s}(p)$, and at most $p$ means $\operatorname{MAX}_{s}(p){ }^{1}$

1 The reader may wonder why we opt to make at least and at most sensitive to the QUD in the style of Beaver \& Clark (2008) rather than picking up focus alternatives as in Rooth 1992. This choice is not crucial, but the Beaver-and-Clark-style framework is slightly easier to work with than Rooth's when it comes to dealing with scales, as Coppock \& Beaver (to appear) discuss. If we were to adopt a Rooth-style approach, a theory of where the strength 
Raising and resolving issues with scalar modifiers

(23) a. $\quad \llbracket$ at least $\rrbracket^{s}=\lambda p \cdot \operatorname{MIN}_{s}(p)$

b. $\llbracket$ at most $\rrbracket^{s}=\lambda p . \operatorname{MAX}_{s}(p)$

Applying the Geach rule as described above gives the following variants:

(24) a. $\llbracket$ at least $\rrbracket^{s}=\lambda \alpha_{\langle\tau, p\rangle} . \lambda \beta_{\tau} . \operatorname{MIN}_{s}(\alpha(\beta))$

b. $\llbracket$ at most $\rrbracket^{s}=\lambda \alpha_{\langle\tau, p\rangle} . \lambda \beta_{\tau} . \operatorname{MAX}_{S}(\alpha(\beta))$

Two virtues of this account should be immediately obvious: It accounts for (i) the inference pattern in (18) showing that only entails at most and presupposes at least, and (ii) the fact that all of these items relate to scales that are ranked by pragmatic strength.

\subsection{Focus-sensitivity}

The analysis in (23)/(24) also accounts for the focus-sensitivity of superlative modifiers. The QUD of the state is systematically related to focus by the Focus Principle: ${ }^{2}$

(25) Focus Principle (Beaver \& Clark 2008)

Some part of a declarative utterance must evoke all of the possibilities in the QUD.

We assume following Rooth $(1985,1992)$ that every expression $\alpha$ has an alternative semantic value $\llbracket \alpha \rrbracket^{A}$; an expression "evokes" a set of alternatives by having that set as its alternative semantic value. The alternative set for an unfocused atomic constituent is the singleton set containing the ordinary semantic value of that constituent. But if a constituent is focused, then the alternative set will be a set of objects that have the same type as the focused constituent. Alternative semantic values are computed recursively in the standard way (essentially as in Rooth 1985). The Focus Principle says that the QUD must be a (possibly non-proper) subset of the alternative semantic value of some part of the utterance $\alpha$ :

$$
\hat{s} \subseteq \llbracket \alpha \rrbracket^{A}
$$

ranking comes from and how it relates to the alternatives would have to be developed. These questions are already answered in the Beaver and Clark framework, where the strength ranking over the salient set of alternatives is provided by the information state.

2 This is quite similar to Rooth's (1992) 'question-answer constraint', which is a special case of his Focus Interpretation Principle. Beaver and Clark's Focus Principle is more general in that it pertains not only to explicit questions but also to implicit questions. 
Note that the QUD may have fewer alternatives than the alternative semantic value of $\alpha$ because the QUD only contains alternatives that are consistent with the common ground, and the alternative semantic value is computed blindly, taking only semantic type into account.

The Focus Principle means that superlative modifiers are correctly predicted to be focus-sensitive on this account (unlike the original generalized quantifier account, but like most subsequent ones). This was illustrated above with (5), repeated here.

(26) a. We should at least invite [the postdoc $]_{F}$ to lunch.

b. We should at least invite the postdoc to [lunch $]_{F}$.

Because the sentences in (26) differ with respect to their focused constituents, they impose different constraints on the QUD via the Focus Principle. The alternatives to (26a) are the propositions expressed by sentences of the form "We should invite $\alpha$ to lunch." This means that (26a) must relate to the question "Who should we invite to lunch?". At least on one interpretation, answers can be ranked according to the importance of the invited individual (Professor Smith, for example, would correspond to a higher-ranked alternative). For (26b), the alternatives are propositions expressed by sentences of the form "We should invite the postdoc to $\alpha$," so the question should be "What should we invite the postdoc to?", with answers ranked by fanciness or expensiveness, dinner being a higher-ranked alternative to lunch. This gives the result that (26a) implies that we should invite someone to lunch, while (26b) does not. Likewise, we predict that (26b) implies that we should invite the postdoc to something, whereas (26a) does not.

\subsection{Distribution}

Now let us consider the syntactic differences between superlative and comparative modifiers, shown above in (9), repeated here as (27). ${ }^{3}$

(27) a. Betty had three martinis \{at most /*fewer than\}.

b. $\quad$ At least /*More than\}, Betty had three martinis.

c. Wilma danced with \{at most /*fewer than\} every second man who asked her.

3 Louise McNally points out that at most does not seem to be quite as polymorphic as at least. For example, She will be at most satisfied with the results does not sound as natural as She will be at least satisfied with the results. This is an unsolved puzzle. 
Raising and resolving issues with scalar modifiers

d. Wilma danced with \{at least /?more than\} Fred and Barney.

According to the definitions of at least and at most given above in (23a) and (23b), they combine with a proposition, which predicts that they can occur clause-peripherally, as in (27a) and (27b).

We also allowed for the possibility that further variants may be derived through applying the Geach rule. This yields items that take two arguments, $\alpha$ and $\beta$, where $\alpha$ is type $\langle\tau, p\rangle$ and $\beta$ is type $\tau$, and $\tau$ can be any type. When $\tau$ is $\langle e, p\rangle$, at least and at most have type $\langle\langle\langle e, p\rangle, p\rangle,\langle\langle e, p\rangle, p\rangle\rangle$, that is, they can modify generalized quantifiers. This analysis can be applied to (27c) and (27d), where it appears that at least and at most are modifying noun phrases whose type is $\langle\langle e, p\rangle, p\rangle$.

Geurts \& Nouwen (2007) propose that comparative modifiers can only modify first-order predicates, i.e., those of type $\langle e, p\rangle$. In our framework, their analysis could be formalized as follows (cf. their definition on p. 540, ex. (31)):

$$
\llbracket \text { more than } \rrbracket^{s}=\lambda P_{\langle e, p\rangle} . \lambda x \cdot \lambda w . \exists P^{\prime}\left[P^{\prime}(x)>_{s} P(x) \wedge P^{\prime}(x)(w)\right]
$$

We hereby add our names to the growing list of researchers on this topic who have endorsed this assumption, and thereby explain the distributional restrictions on comparative modifiers.

\subsection{Truth conditions}

One very fundamental virtue of this account is that it correctly captures the truth conditions of sentences with at least. (This virtue is crucially not shared by Geurts \& Nouwen's (2007) account, as Cohen \& Krifka (2011) point out.) Consider (29), with focus on the numeral. This sentence is false if John petted exactly two rabbits.

(29) John petted at least three rabbits.

In order to evaluate whether or not our analysis makes the right predictions regarding this case, we must make some assumptions about the meanings of numerals. Since Horn (1972), the dominant view has been that numerals have what Horn (1992) calls a 'one-sided' meaning, so that e.g. Two pens are on the table is true (though infelicitous) if there are three pens on the table. Others (see Kennedy 2012 for a recent summary) have argued for a 'two-sided' analysis, on which two pens is semantically equivalent to exactly 
two pens. However, even Kennedy (2012), a proponent of the 'two-sided' view, admits that numerals may be ambiguous, having both one-sided and two-sided readings.

To be on the safe side, we should show that our analysis delivers the right truth conditions regardless of what we assume regarding numerals. Let us first consider a two-sided ('exactly') analysis for three. Let $p_{n}$ stand for the set of worlds where John petted exactly $n$ rabbits. Given that focus is on three, our question in $s$ should be how many rabbits John petted, with answers ranked by number of petted rabbits $\left(p_{0}<_{s} p_{1}<_{s} p_{2}<_{s} \ldots\right)$. The denotation of John petted at least three rabbits will then be as follows, speaking set-theoretically:

\section{$\llbracket J o h n$ petted at least three rabbits $\rrbracket^{s}=\bigcup\left\{p_{n} \mid n \geq 3\right\}$}

The worlds in which exactly two rabbits were petted by John are not in the denotation of the sentence, as desired. We get the same result if we let $p_{n}$ stand for the set of worlds where John petted $n$ or more rabbits. Hence regardless of what stance we take on the analysis of numerals, we correctly derive the result that John petted at least three rabbits is false in worlds where John petted only two.

The corresponding sentence with at most should be false in a world where John petted four rabbits. The definition in terms of MAX ensures that the denotation of John petted at most three rabbits includes no worlds that are included in an alternative ranked higher than John petted three rabbits. Let us begin with a two-sided analysis of numerals. Let $p_{n}$ stand for the set of worlds where John petted exactly $n$ rabbits, and let $\omega$ stand for the set of all worlds. The denotation of John petted at most three rabbits is, in set theoretical terms:

(31) 『John petted at most three rabbits $\rrbracket^{s}=\omega-\bigcup\left\{p_{n} \mid n>3\right\}$

The denotation excludes all of the worlds where John petted exactly four rabbits. This result also follows if we use a one-sided analysis of numerals: Let $p_{n}$ now stand for the set of worlds where John petted $n$ or more rabbits. The denotation of our sentence can again be expressed with the formula in (31). All of the worlds in $p_{4}$ are eliminated, which means that all of the worlds where John petted four or more rabbits are not in the denotation. Thus regardless of our assumptions about numerals, we correctly predict that John petted at most three rabbits is false if John petted four rabbits. 
Raising and resolving issues with scalar modifiers

Now, suppose that John did in fact pet exactly four rabbits. According to our analysis, John petted at least three rabbits is true, and John petted at most three rabbits is false. This accords with our intuitions, and those of Cohen \& Krifka (2011: pp. 6-7), but it is controversial whether this is a bug or a feature. Geurts \& Nouwen (2007) and Geurts, Katsos, et al. (2010) show that native speakers are not reliably willing to agree that John petted four rabbits implies John petted at least three rabbits, or John petted at most five rabbits. Approximately half of the native speakers of Dutch that they asked judged both of these inferences to be invalid (in Dutch). These results do not establish that they are in fact semantically invalid, because superlative modifiers convey more pragmatic information than the sentence with the bare numeral, namely ignorance implicatures. As Kaplan (1999) discusses, validity intuitions are affected by whether the information conveyed by the conclusion is contained in the information conveyed by the premise, and truth-conditions are not the only relevant kind of information that may play a role in such judgments. On the other hand, the fact that $50 \%$ of the respondents did see these as valid inferences suggests that these inferences are somehow valid, and indeed under our proposal, these inferences are logically valid despite being pragmatically invalid. We therefore see the prediction that they are semantically valid as a feature, as long as an explanation for why they are pragmatically invalid comes with the theory as well. We will offer an explanation in $\S 3$.

Because 'at most two' entails 'at most three' according to our proposal, we predict that the following inference is valid:

(32) a. If Berta has had at most three drinks, she is fit to drive. Berta has had at most two drinks.

b. Berta is fit to drive.

This is in line with our intuitions, as well as those of linguistically untrained native speakers of English, according to experimental results obtained by Cummins \& Katsos (2010). Crucially, this inference is predicted to be invalid under Geurts \& Nouwen's (2007) theory, where superlatives are defined in terms of the epistemic state of the speaker, as we discuss in further detail in $\S 4$.

Furthermore, unlike Geurts \& Nouwen's (2007) theory, the present analysis is extensional. We agree with Cohen \& Krifka (2011) that this is a virtue. Suppose that, in world $w$, all rabbits are magical beings, and there are no other magical beings in $w$. Then (29) has the same truth value as (33) in $w$ : 
(33) John petted at least three magical beings.

This equivalence holds regardless of whether or not anybody is aware of the magical nature of rabbits, as Cohen \& Krifka (2011) point out. Some speakers may hold one of these statements to be true while failing to hold the other to be true, but this would stem from faulty information regarding the state of the world, and one of these two beliefs would be mistaken. If indeed rabbits are magical, then Mary petted at least three magical beings if she petted at least three rabbits, regardless of what anybody believes about rabbits.

\subsection{Relation to Büring's theory}

We should note at this point that our analysis of at least is quite similar to Büring's (2008) proposal, which builds in turn very much on Krifka 1999. Recognizing that alternatives may be ranked in a fashion that does not correspond to entailment, Krifka (1999) introduces a strength ranking over focus alternatives, and defines the meaning of at least $\alpha$ as the semantic union over the focus alternatives ranked as high as or higher than $\alpha$. Büring (2008) uses this idea and construes the meaning of at least in terms of disjunction.

Büring (pp. 118-119) defines at least in combination with a propositiondenoting expression $\alpha$ syncategorematically as follows (we are copying his notation verbatim here):

（34） a. $\quad \llbracket$ at least $\alpha \rrbracket=[\llbracket \alpha \rrbracket-\bigcup(\operatorname{ABOVE}(\alpha))] \vee \bigcup(\operatorname{ABOVE}(\alpha))$

b. $\operatorname{ABOVE}(\alpha)=\bigcup\left\{O^{\prime} \mid\left\langle\llbracket \alpha \rrbracket, O^{\prime}\right\rangle \in \llbracket \alpha \rrbracket^{A}\right\}$

$\llbracket \alpha \rrbracket^{A}$ is the alternative semantic value of $\alpha$, but here, it is not a set of alternative values, but rather a strength relation over alternative values, following Krifka (1999). If $\langle a, b\rangle$ is a member of this relation, then $b$ is "above" $a$. The elements of the alternative semantic value here are likewise pairs of values such that the second is stronger than the first.

If alternative semantic values are ranked as Krifka proposes, the Focus Principle should require congruence between the alternative semantic values and the strength ranking, rather than the QUD. Here is a version of the Focus Principle that does that:

\section{(35) Scalar Focus Principle}

A declarative utterance in context $s$ must contain an expression $\alpha$ such that $\llbracket \alpha \rrbracket^{A} \subseteq \geq_{s}$ 
Raising and resolving issues with scalar modifiers

Armed with this principle, we can recast Büring's lexical entry for at least as stated in (34) non-syncategorematically as in (36):

(36) $\llbracket$ at least $\rrbracket^{s}=\lambda p . \lambda w . \exists q\left[q \geq_{s} p \wedge q(w)\right]$

And this is exactly what we have as the definition of MIN. Hence, for all $p$ of type $\langle s, t\rangle$, and for all $w$ :

$$
\llbracket \text { at least } \rrbracket^{s}(p)(w) \Longleftrightarrow \operatorname{MIN}_{s}(p)(w)
$$

Thus our temporary proposal for at least can be seen as a cross-categorial and non-syncategorematic variant of Büring's proposal, based on a slightly different treatment of focus alternatives. (Büring does not offer an analysis of at most, which we do here.)

As we have just argued, this solution has a number of advantages. But we will argue in \$3.1 that Büring’s analysis suffers from a conceptual problem that prevents it from being able to explain the implicature data. To remedy this problem and account for this data, we enrich the analysis with inquisitive semantics.

\section{At least in inquisitive semantics}

\subsection{Problem: Ignorance implicatures}

What we have said so far predicts I read at least three books to be equivalent to I read more than two books, both semantically and pragmatically. But recall (7), repeated here as (37).

(37) a. \#A hexagon has at least five sides.

b. A hexagon has more than four sides.

Most people know how many sides a hexagon has. So for most people, (37a) is infelicitous, because it implies that they do not know this. On the other hand, (37b) seems not to give rise to the same ignorance implicature.

Nothing we have said so far explains this contrast between superlative and comparative modifiers. Our theory thus far is basically equivalent to Büring's, and although Büring purports to explain this contrast, his explanation is conceptually problematic. According to Büring, at least "amounts to" a disjunction between the prejacent and its higher-ranked alternatives. This means, according to Büring, that it is subject to the following implicature schema: 
(38) If a speaker utters $p$ or $q$, it is implied that (i) in all of the speaker's doxastic alternatives $p \vee q$ and (ii-a) in some of the speaker's doxastic alternatives $p$, and (ii-b) in some $q$. Büring (2008: 114f)

In what sense is at least disjunctive on Büring's account? One might point to the disjunction symbol $\vee$ on the right-hand side of the equals sign in (34), and say that this is sufficient to establish the point. The disjunction symbol is part of the meta-language description of the meaning of at least, so at least expresses a disjunction, one might reason. (The symbol $\vee$ is not usually used to represent a binary set operation, but in this expression, its two arguments must be sets, since they themselves are the result of union or set complementation operations. We can make sense of this notation by assuming that $\vee$ is meant to represent union.)

Yet the fact that the meta-language description of the meaning contains a disjunction symbol does not mean that the expression is disjunctive in any sense that we can hang implicatures on. We cannot hang our theory of implicature on the meta-language description of the meaning, saying, "If the meta-language description of the meaning of a sentence contains a symbol representing disjunction, then a speaker who utters that sentence should consider both of the disjuncts possible." Speakers do not have conscious or subconscious access to how linguists happen to formulate the description of denotations. And the putative disjunctiveness of the expression cannot be determined by looking at the set of possible worlds where the sentence is true, so it is not at the level of denotation that the sentence is disjunctive. Sentences containing at least are obviously not disjunctive on the level of surface form, either. Büring does not describe any covert syntactic transformation that would turn at least sentences into disjunctions at LF, so it is not at LF that they are disjunctive either, on his analysis. The only level at which at least sentences are disjunctive on Büring's analysis is in the meta-language description of the meaning.

Now, we might suppose that there is a covert syntactic transformation which transforms the at least sentence into a disjunction at LF. Then we could generate an ignorance implicature by interpreting the implicature schema as follows: "If the LF of a sentence contains a disjunction, then the speaker should consider both of the propositions expressed by the two disjuncts possible." This interpretation of the implicature schema is reasonable, and it would obviously work for sentences with the surface form of a disjunction, assuming that they are also disjunctions at LF. This is distinct from what 
Raising and resolving issues with scalar modifiers

Büring states, though; he does not describe a syntactic transformation, but rather expresses the meaning of an at least sentence using a disjunction in the meta-language. Some other theorist might choose to pursue and defend the idea of such a syntactic transformation, but we find it implausible that sufficient empirical motivation for such a drastic alteration of the syntax could be found.

Alternatively, suppose that rather than stating the meanings of natural language expressions directly as we have been doing, we translate natural language expressions into a formal language. This is what Montague (1974b) did in 'The Proper Treatment of Quantification in Ordinary English' (PTQ): Rather than giving semantics for English directly as he did in 'English as a Formal Language' (Montague 1974a), Montague (1974b) used Intensional Logic (IL) as an intermediate step in deriving truth conditions for English in PTQ, specifying a translation procedure from English to IL, and letting the semantics of the natural language English effectively be inherited from the semantics of the formal language IL. With such a set-up, one might imagine hanging the theory of implicature on the syntactic form of the formal language into which English is translated, saying "If the translation of $\alpha$ is of the form ' $\phi \vee \psi$ ', then ...". This does not seem to be what Büring intends, given both his use of $\llbracket \cdot \rrbracket$, which standardly signifies denotation rather than translation, and his use of set-theoretic notation on the right-hand side of the equals sign, which is usually part of the meta-language in formal semantics. Furthermore, Montague made it very clear that the translation procedure was only meant to be a convenience, and that one should always be able to state the denotations of natural language expressions directly, dispensing with IL. Some other theorist may dare to hang implicatures on the syntax of a translation in spite of this. We, however, choose a different way out of the problem.

If we turn to inquisitive semantics, then we can give substance to the notion that at least sentences have something in common with disjunctions, without resorting to the claim that there is any level of representation at which they are disjunctions. Our proposed solution for both of these problems is to treat at least as an inquisitive semantics-style proposition including the prejacent and the higher-ranked possibilities. More specifically, at least $p$ denotes the set containing all possibilities $p^{\prime}$ such that $p^{\prime}$ is at least as strong as $p$ according to the pragmatic strength ranking over answers to the QUD. 


\subsection{Framework}

In a classical semantic setting, the denotation of a declarative sentence is a set of possible worlds, and the denotation of a question is a set of sets of possible worlds. In inquisitive semantics, declarative sentences and interrogative sentences both denote sets of sets of possible worlds. Sets of possible worlds are called possibilities, and the word 'proposition' is used for sets of possibilities. In the 'unrestricted' version of inquisitive semantics (Ciardelli, Groenendijk \& Roelofsen 2009, 2012), which we adopt here, a proposition is defined as follows:

\section{(39) Proposition}

A proposition is a non-empty set of possibilities $P$ such that either:

(i) $\emptyset \notin P$, or

(ii) $P=\{\emptyset\}$.

In contrast to the original variant of inquisitive semantics (Groenendijk \& Roelofsen 2009), this means that propositions may non-trivially contain non-maximal possibilities, i.e., possibilities that are contained within other possibilities. This will play an important role for our analysis of superlative modifiers.

Inquisitive semantics is typically defined for the language of classical logic, but here we use a Montague-style (Montague 1974a, not Montague 1974b) semantics in which natural language expressions are interpreted directly, with unrestricted inquisitive-type semantic values, following Roelofsen \& van Gool (2010). Here are some basic lexical entries, which are simply singleton sets containing the usual denotation.

(40) $\llbracket A n n \rrbracket=\{A n n\}$

(41) $\llbracket$ Bill $\rrbracket=\{$ Bill $\}$

(42) $\llbracket$ snores $\rrbracket=\{\lambda x . \lambda w \cdot x$ snores in $w\}$

Following Roelofsen \& van Gool (2010), we assume that the meanings of expressions are put together through a pointwise version of functional application (a.k.a. "Hamblin Functional Application", from Hamblin 1973).

(43) Pointwise Function Application

If $\llbracket \alpha \rrbracket \subseteq D_{\langle\sigma, \tau\rangle}$ and $\llbracket \beta \rrbracket \subseteq D_{\sigma}$, then

$\llbracket \alpha \beta \rrbracket=\llbracket \beta \alpha \rrbracket=\left\{d \in D_{\tau} \mid \exists a \in \llbracket \alpha \rrbracket: \exists b \in \llbracket \beta \rrbracket: d=a(b)\right\}$ 
Raising and resolving issues with scalar modifiers

For example:

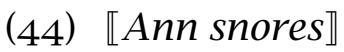

$=\{\lambda w$. Ann snores in $w\}$

$=\left\{\left\{w_{10}, w_{11}\right\}\right\}$

To be pedantic: This is obtained from Pointwise Function Application with

$\alpha=$ snores and $\beta=A n n, \sigma=e$ and $\tau=p . \llbracket \alpha \rrbracket \subseteq D_{\langle e, p\rangle}$ because it is a singleton set containing one element of that type (a property) and $\llbracket \beta \rrbracket \subseteq D_{e}$ because it is a set containing one individual. The result is the set containing the unique proposition obtainable by pairing the property with the individual.

To see the expressive power of inquisitive semantics, consider disjunction as treated in Roelofsen \& van Gool 2010.

\section{(45) Disjunction}

For any type $\tau$, if $\llbracket \alpha \rrbracket, \llbracket \beta \rrbracket \subseteq D_{\tau}$, then $\llbracket \alpha$ or $\beta \rrbracket=\llbracket \alpha \rrbracket \cup \llbracket \beta \rrbracket$

Hence:

(46) $\llbracket$ Ann or Bill $\rrbracket=\{$ Ann, Bill $\}$

(47) «Ann or Bill snores》

$=\{[\lambda w$. Ann snores in $w],[\lambda w$. Bill snores in $w]\}$

$=\left\{\left\{w_{10}, w_{11}\right\},\left\{w_{01}, w_{11}\right\}\right\}$

In inquisitive semantics, disjunctions denote sets containing multiple possibilities. A classical analysis of disjunction, in contrast, would treat Ann or Bill snores as an undifferentiated set of possible worlds. The contrast between classical and inquisitive disjunction is depicted in Figure 1.

Following Ciardelli, Groenendijk \& Roelofsen (2009), we can define inquisitive as follows:

(48) Inquisitivity (Ciardelli, Groenendijk \& Roelofsen 2009)

A sentence $\phi$ is inquisitive iff $\llbracket \phi \rrbracket$ contains at least two maximal possibilities.

A maximal possibility is one that is not fully contained by any other possibility. Ann or Bill snores is inquisitive in this sense, because its denotation contains two maximal possibilities.

The union of all of the possibilities in a proposition is its informational content. A sentence is informative if its informational content does not cover 


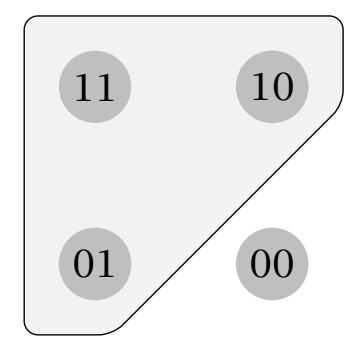

(a) Classical

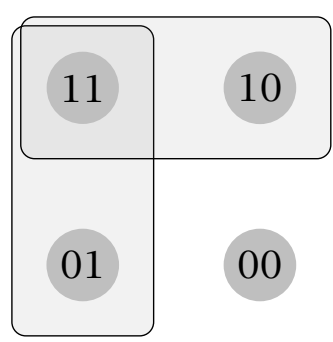

(b) Inquisitive

Figure 1 Classical and inquisitive disjunction

the whole space of possible worlds. Formally (letting $\omega$ stand for the set of all possible worlds):

(49) Informativity (Ciardelli, Groenendijk \& Roelofsen 2009) $\phi$ is informative iff $\bigcup \llbracket \phi \rrbracket \neq \omega$

Thus Ann or Bill snores is informative, assuming that $\omega$ contains a world where neither Ann nor Bill snores. The yes/no question Does Ann snore? on the other hand would denote a partition on the full space of possible worlds, and hence be inquisitive but not informative. Ann snores is informative but not inquisitive.

A proposition that contains exactly two possibilities, where one is a subset of the other, is not inquisitive, even though it contains multiple possibilities, because there is only one maximal possibility. However, such a proposition is attentive.

(50) Attentivity (Ciardelli, Groenendijk \& Roelofsen 2009)

$\phi$ is attentive iff $\llbracket \phi \rrbracket$ contains a non-maximal possibility.

The idea behind this label is that one draws attention to all of the possibilities in the proposition that one puts forth into a discourse. For example, according to Ciardelli, Groenendijk \& Roelofsen (2009), might $p$ draws attention to $p$, but does not provide any new information, and this is modelled by treating might $p$ as the proposition containing $p$ and the 'trivial possibility' consisting of all worlds.

For the discussion of superlative modifiers, it will be useful to have a cover term for both of the ways in which propositions in inquisitive semantics may 
Raising and resolving issues with scalar modifiers

be more fine-grained than those of the classical setting. We use interactive for this purpose, and define it as follows:

\section{(51) Interactivity}

$\phi$ is interactive iff $\llbracket \phi \rrbracket$ contains more than one possibility.

It follows from this definition that $\phi$ is interactive if and only if it is either inquisitive or attentive. As we will see in the next section, sentences containing superlative modifiers are, in general, interactive in this sense.

\subsection{Superlative modifiers}

For superlative modifiers, the valuation function needs to be parameterized by a state containing the QUD and the ranking over the answers, as we had in the previous section. We propose that, in a state $s$, at least $\alpha$ denotes the set of possibilities $p$ such that $p$ is stronger according to $s$ than some possibility in $\llbracket \alpha \rrbracket^{s}$. We can thus state the meaning of at least syncategorematically as follows:

\section{(52) 【at least $\alpha \rrbracket^{s}=\left\{p \mid p \geq_{s} p^{\prime}\right.$ for some $\left.p^{\prime} \in \llbracket \alpha \rrbracket^{s}\right\}$}

In order to arrive at this result compositionally through Pointwise Functional Application, we can define the meaning of at least as a set of functions from possibilities to possibilities, where the output of each function is some possibility that is pragmatically stronger than the input possibility. We use choice functions to formalize this. ${ }^{4}$ A choice function is a function which, applied to a non-empty set, returns some member of that set. (We may assume that the empty set is not in the domain of a choice function.) The propositional version of at least can be defined as the following set of $\langle p, p\rangle$ functions:

(53) $\llbracket$ at least $\rrbracket^{s}=\left\{\lambda p . f\left\{p^{\prime} \in \hat{s} \mid p^{\prime} \geq_{s} p\right\} \mid f\right.$ is a choice function $\}$

To generalize this, as above, we allow at least to combine first with any argument whose type ends in $p$, and next with anything that will "complete" the first argument to make a proposition. If $\alpha$ is a function of type $\langle\sigma, p\rangle$ and $\beta$ is a function of type $\sigma$, then:

4 Thanks to an anonymous reviewer for pointing out a problem with how the analysis was formalized in a previous draft and suggesting this alternative. 
(54) $\llbracket$ at least $\rrbracket^{s}=\left\{\lambda \alpha . \lambda \beta . f\left\{p \in \hat{s} \mid p \geq_{s} \alpha(\beta)\right\} \mid f\right.$ is a choice function $\}$

Let us apply this to the following example:

(55) At least $[\mathrm{Ann}]_{F}$ snores.

The focus-marking on Ann should ensure that the question under discussion concerns who snores. We may assume that the possible answers are $a$ 'Ann snores', $b$ 'Bill snores', and $a \& b$ 'Ann and Bill snore', the last being ranked above the first two, as in §2.1.

In order to use our lexical entry with a proper name like Ann, we must lift the proper name into a generalized quantifier. Since we are working in an inquisitive framework, the value should be the set containing that generalized quantifier. So let us assume:

$$
\llbracket A n n \rrbracket=\left\{\lambda P \in D_{\langle e, p\rangle} . P(\mathrm{Ann})\right\}
$$

Combining this with at least via Pointwise Functional Application gives us:

(57) $\llbracket$ at least Ann $\rrbracket^{s}=\left\{\lambda P . f\left\{p \in \hat{s} \mid p \geq_{s} P(\right.\right.$ Ann $\left.)\right\} \mid f$ is a choice function $\}$

Combining this with our lexical entry for snores gives us the result in (58).

(58) $\llbracket$ At least Ann snores $\rrbracket^{s}$

$=\left\{f\left\{p \in \hat{s} \mid p \geq_{s} a\right\} \mid f\right.$ is a choice function $\}$

$=\left\{p \in \hat{s} \mid p \geq_{s} a\right\}$

$=\{a, a b\}$

$=\left\{\left\{w_{10}, w_{11}\right\},\left\{w_{11}\right\}\right\}$

The denotation of At least Ann snores is depicted on the righthand panel of Figure 2, which also shows the denotation of Ann snores for comparison on the left.

As Figure 2 illustrates, At least Ann snores and Ann snores have the same informational content. But the at least sentence is interactive. This difference can explain why sentences with superlative modifiers give rise to ignorance implicatures while corresponding sentences with comparative ones do not, as we explain in the next section. In the section after that, we will show that this assumption can also explain why Ann snores gives rise to an exhaustivity implicature while At least Ann snores does not.

In the case where the scale over the answers to the QUD is not an entailment scale, and higher-ranked answers entail lower-ranked answers, at least has a truth conditional effect, and is inquisitive but not attentive. 
Raising and resolving issues with scalar modifiers
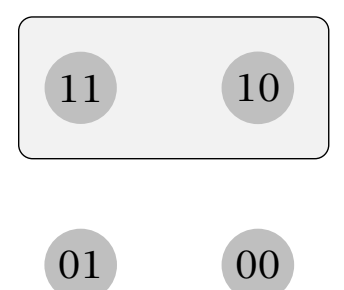

(a) Ann snores
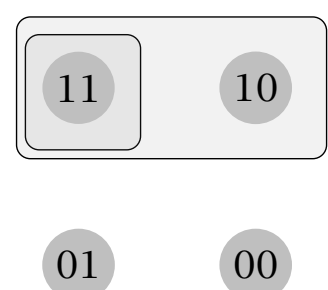

(b) At least Ann snores

Figure 2 Attentive and non-attentive propositions

(59) a. Ann is an assistant professor.

b. Ann is at least an assistant professor.

Example (59a) is false if Ann is a full professor; example (59b) is not, assuming that the QUD concerns Ann's academic rank, and the answers are ranked according to prestige. Inserting at least can affect the truth conditions in this context because the higher-ranked alternatives in such a context do not entail the lower-ranked alternatives and therefore widen the informational content. In such cases, there are multiple maximal possibilities, so (59b) is inquisitive. Assuming that none of the alternatives are nested within each other, (59b) is not attentive, unlike At least Ann snores. Thus in general, sentences with superlative modifiers are interactive, and may be either inquisitive or attentive.

At most is a tiny bit more complicated than at least. If we treat at most as the set of all of the alternatives weaker than or as strong as the prejacent, then we will lose the correct truth conditions that we had under the treatment using MAX. At most Ann smokes would not end up being false if both Ann and Bill smoke, because it would simply denote the set containing the proposition that Ann smokes, which does not rule out the possibility that Bill smokes. The MAX treatment from the previous section excludes all worlds that are found in a higher-ranked alternative from the informational content. To capture this negative character of at most, we propose the following, continuing to use the same definition of MAX:

(6o) $\llbracket$ at most $\rrbracket^{s}=\left\{\lambda \alpha . \lambda \beta . f\left\{p \cap \operatorname{MAX}_{s}(\alpha(\beta)) \mid p \leq_{s} \alpha(\beta)\right\}\right.$

$f$ is a choice function $\}$ 
So At most Ann smokes will denote:

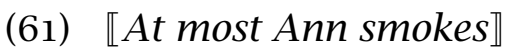

$=\left\{f\left\{p \cap \operatorname{MAX}_{s}(a) \mid p \leq_{s} a\right\} \mid f\right.$ is a choice function $\}$

$=\left\{p \cap \operatorname{MAX}_{s}(a) \mid p \leq_{s} a\right\}$

$=\left\{p \cap\left\{w_{10}\right\} \mid p \leq_{s}\left\{w_{10}, w_{11}\right\}\right\}$

$=\left\{p \cap\left\{w_{10}\right\} \mid p \in\left\{\left\{w_{10}, w_{11}\right\}\right\}\right\}$

$=\left\{\left\{w_{10}, w_{11}\right\} \cap\left\{w_{10}\right\}\right\}$

$=\left\{\left\{w_{10}\right\}\right\}$

$\operatorname{MAX}(a)$ is the set of worlds that are not contained in any alternative ranked higher than (or unranked with respect to) $a$. So in the current example, $\operatorname{MAX}(a)$ is $\left\{w_{10}\right\}$, the set containing the worlds where Ann smokes and Bill does not. There is only one proposition $p$ that is weaker than or as strong as $a$, namely $a$, so we have only one $p$ to consider. Taking the intersection of $a=\left\{w_{11}, w_{10}\right\}$ and $\left\{w_{10}\right\}$ gives us $\left\{w_{10}\right\}$. Thus the denotation of At most Ann smokes on this system is $\left\{\left\{w_{10}\right\}\right.$. Since Ann smokes is the lowest-ranked alternative, the denotation does not turn out to be interactive in this example. But in general, at most sentences will be interactive.

To see how this analysis of at most works with an entailment scale, let us consider the following example under a two-sided analysis of numerals.

(62) At most three bananas are ripe.

Let $p_{n}$ stand for the proposition that exactly $n$ bananas are ripe. For the sake of discussion, suppose there is exactly one world for every number of bananas that are ripe, so $w_{n}$ is the (single) world where exactly $n$ bananas are ripe. So for all $n, p_{n}=\left\{w_{n}\right\}$. The question is how many bananas are ripe, so $\hat{s}=\left\{p_{n} \mid n \geq 0\right\}$. Answers are ranked by number of bananas, so $p_{n}>_{s} p_{m}$ if and only if $n>_{s} m$. The denotation of (62) is as follows:

(63) $\llbracket$ At most three bananas are ripe $\rrbracket=\left\{p \cap \operatorname{MAX}_{s}\left(p_{3}\right) \mid p \leq_{s} p_{3}\right\}$

Set-theoretically speaking, $\operatorname{MAX}_{s}\left(p_{3}\right)$ is the set of worlds $w$ in $\omega$ that are not included in any possibility ranked higher than $p_{3}$. This is the set $\left\{w_{0}, w_{1}, w_{2}, w_{3}\right\}$. This set will be intersected with all of the propositions that are ranked lower than or as high as $p_{3}$, namely $p_{0}-p_{3}$. All of these propositions are subsets of $\operatorname{MAX}_{s}\left(p_{3}\right)$, so taking the intersection will not have any effect, and the resulting denotation will be $\left\{p_{0}, p_{1}, p_{2}, p_{3}\right\}$. In general, 
Raising and resolving issues with scalar modifiers

when the answers to the QUD are disjoint, taking the intersection with MAX will have no effect.

For comparison, consider (62) under a one-sided analysis of numerals. Let us now use $p_{n}$ to denote the possibility that $n$ or more bananas are ripe, so $p_{3}=\left\{w_{3}, w_{4}, w_{5}, \ldots\right\}$. We still assume that $\hat{s}=\left\{p_{n} \mid n \geq 0\right\}$, and $p_{n}>_{s} p_{m}$ if and only if $n>m$. $\operatorname{MAX}_{s}\left(p_{3}\right)$ is the set of worlds $w$ in $\omega$ that are not included in any possibility ranked higher than $p_{3}$. Again, this turns out to be the set $\left\{w_{0}, w_{1}, w_{2}, w_{3}\right\}$. And again, this set will be intersected with all of the propositions that are ranked lower than or as high as $p_{3}$, namely $p_{0}-p_{3}$. However, in this case, taking the intersection with MAX will make a difference.

$$
\begin{aligned}
& \llbracket \text { At most three bananas are ripe } \rrbracket \\
& =\left\{p \cap \operatorname{MAX}_{s}\left(p_{3}\right) \mid p \leq_{s} p_{3}\right\} \\
& =\left\{p \cap\left\{w_{0}, \ldots, w_{3}\right\} \mid p \in\left\{p_{0}, \ldots, p_{3}\right\}\right\} \\
& =\left\{\left\{w_{0}, \ldots, w_{3}\right\},\left\{w_{1}, w_{2}, w_{3}\right\},\left\{w_{2}, w_{3}\right\},\left\{w_{3}\right\}\right\}
\end{aligned}
$$

So the denotation under the one-sided analysis is not $\left\{p_{0}, p_{1}, p_{2}, p_{3}\right\}$, as we had under the two-sided analysis. Rather, it is a proposition in which each one of these possibilities has been shrunk to exclude possibilities ranked higher than the prejacent. This gives us the right truth conditions, regardless of how numerals are interpreted.

\subsection{Explaining the implicatures}

\subsubsection{Ignorance}

Intuitively, the reason that At least Ann snores conveys an ignorance implicature is that the speaker is drawing attention to the issue of whether Ann or anyone else snores by uttering this sentence, and it doesn't make sense to draw attention to an issue if you already know how to settle it. This intuition can be formalized with the notion of interactive sincerity.

The first ingredient of the explanation is the idea of the information set of a speaker. This is the set of worlds that are epistemically accessible to the speaker. To check whether a given issue is settled in the speaker's information set, one can consider the issue restricted to that set of worlds.

\section{(65) Restriction}

If $k$ is an information set (set of possible worlds) and $P$ is an inquisitivestyle proposition, then $P$ restricted to $k, P \uparrow k$ is:

$P \vdash k=\operatorname{PRO}\{p \mid \exists q \in P: p=k \cap q\}$ 
where $\operatorname{PRO}(P)$ ensures that $P$ is a proposition. We assume the following definition of propositional closure.

(66) Propositional closure (Ciardelli, Groenendijk \& Roelofsen 2009)

$$
\operatorname{PRO}(P)= \begin{cases}\{\emptyset\} & \text { if } P=\{\emptyset\} \\ P-\{\emptyset\} & \text { otherwise }\end{cases}
$$

(Nothing under discussion here hinges on this as far as we can see; we adopt this only to ensure that we use the term 'proposition' consistently.)

Now the notion of interactiveness of an expression relative to a state can be defined:

\section{(67) Interactiveness in a state}

$\phi$ is interactive in an information set $k$ iff $\llbracket \phi \rrbracket \mid k$ contains more than one possibility.

The Maxim of Interactive Sincerity requires that if a speaker draws attention to multiple possibilities, then the issue is not entirely settled in his own mind, so to speak.

\section{(68) Maxim of Interactive Sincerity}

If $\phi$ is interactive, then $\phi$ is interactive in the speaker's information set.

This is a generalization of the Maxim of Inquisitive Sincerity as defined by Groenendijk \& Roelofsen (2009), which has inquisitive where we have interactive. ${ }^{5}$ Notice that it is much more general than Büring's implicature schema (38). Büring's implicature schema applies only to disjunctions, while the present principle applies to all kinds of interactive content.

Suppose that the speaker knows that Ann snores and nobody else does (hence Bill does not). Then the speaker's information set is $\left\{w_{10}\right\}$, the set of worlds where that is true (a singleton set in this tiny example). The denotation of At least Ann snores restricted to that state gives a proposition consisting of the state itself. Formally this works as follows:

(69) a. $\quad \llbracket$ At least Ann snores $\rrbracket=\left\{\left\{w_{11}, w_{10}\right\},\left\{w_{10}\right\}\right\}=P$

5 Pruitt \& Roelofsen (2011) also have a sincerity maxim that applies to attentive content, called the Maxim of Attentive Sincerity: Every possibility that $\phi$ draws attention to must be a 'live' possibility for the speaker, i.e., every possibility for $\phi$ must be consistent with the speaker's information set. This would not serve to explain the ignorance implicature in the case of entailment scales, because it would allow that the speaker's information set is contained within the strongest possibility. 
Raising and resolving issues with scalar modifiers

$$
\begin{array}{ll}
\text { b. } & \left\{w_{10}\right\}=k \\
\text { c. } & P \vdash k \\
& =\left\{\left\{w_{11}, w_{10}\right\},\left\{w_{10}\right\}\right\} \vdash\left\{w_{10}\right\} \\
& =\operatorname{PRO}\left\{p \mid \exists q \in\left\{\left\{w_{11}, w_{10}\right\},\left\{w_{10}\right\}\right\}: p=\left\{w_{10}\right\} \cap q\right\} \\
& =\operatorname{PRO}\left\{\left\{w_{10}\right\}\right\} \\
& =\left\{\left\{w_{10}\right\}\right\}
\end{array}
$$

This proposition is not interactive, so Interactive Sincerity is violated.

In general, if the speaker knows Only $P$, then she already knows which of the possibilities expressed by At least $P$ holds, namely $P$. We thus derive the result that only when the speaker's information set is consistent with higherranked alternatives can an at least sentence satisfy Interactive Sincerity. Hence the ignorance implicature.

Now let us consider the hexagon example. Recall (37) from above, repeated again as (70):

(70) a. \#A hexagon has at least five sides.

b. A hexagon has more than four sides.

We are now in a position to account for the fact that (70a) is infelicitous when spoken by someone who knows how many sides hexagons have, while (7ob) is fine in such a situation.

Let us begin by showing how it works using a two-sided analysis of numerals. Suppose the proposition $p_{n}$ is the set of worlds where hexagons have exactly $n$ sides. Our question in $s$ is how many sides hexagons have, with answers ranked by number of sides. For example, $p_{2}$ is ranked below $p_{3}$ and $p_{4}$. Then:

\section{(71) $\llbracket A$ hexagon has at least five sides $\rrbracket^{s}=\left\{p_{n} \mid n \geq 5\right\}$}

The information set of speakers who know how many sides hexagons have is included in $p_{6}$. Crucially, if we restrict the set of possibilities to worlds in $p_{6}$, we will end up with a singleton set: $\left\{p_{6}\right\}$. Hence Interactive Sincerity is violated when the speaker knows how many sides a hexagon has.

We obtain the same result under a one-sided analysis of numerals. Let $p_{n}$ be the set of worlds where hexagons have $n$ or more sides, and let $w_{n}$ be the unique world (for the sake of discussion) where hexagons have exactly $n$ sides. The QUD should consist of a set of nested possibilities $\left\{p_{n} \mid n>0\right\}$. $A$ hexagon has at least five sides will denote $\left\{p_{n} \mid n \geq 5\right\}$. The speaker's 
information set consists only of worlds where hexagons have exactly six sides, i.e. $\left\{w_{6}\right\}$. Intersecting this information set with $p_{7}$ or any other proposition ranked higher than $p_{6}$ yields the empty set. Intersecting it with $p_{5}$ yields $\left\{w_{6}\right\}$, as does intersecting it with $p_{6}$. This means that, restricted to the speaker's information set, the denotation of the sentence is $\left\{\left\{w_{6}\right\}\right\}, \mathrm{a}$ singleton set. This is not interactive, so Interactive Sincerity is violated.

Now for (7ob). We claim that comparative modifiers are not interactive and therefore not subject to Interactive Sincerity. To adapt the lexical entry for more than given in (28) to our inquisitive setting, we simply place that denotation into a singleton set:

(72) $\llbracket$ more than $\rrbracket^{s}=\left\{\lambda P_{\langle e, p\rangle} . \lambda x . \lambda w . \exists P^{\prime}\left[P^{\prime}(x)>_{s} P(x) \wedge P^{\prime}(x)(w)\right]\right\}$

(We are by no means committed to this particular analysis of comparative modifiers; we claim only that their analysis in inquisitive semantics should be a singleton set consisting of whatever the best non-inquisitive analysis is.) After composing with the other elements of the sentence in the usual way, the denotation of (7ob) will end up a singleton set.

\section{(73) $\llbracket A$ hexagon has more than four sides $\rrbracket^{s}$}

$=\{\{w \mid$ a hexagon has more than four sides in $w\}\}$

$=\left\{\left\{w_{5}, w_{6}, \ldots\right\}\right\}$

This proposition is neither inquisitive nor attentive, so it is not subject to Interactive Sincerity. Notice further that the informative content of (70a) and (7ob) is the same: In both cases it is $\bigcup\left\{p_{n} \mid n \geq 5\right\}$. In general, at least $n$ and more than $n-1$ have the same informative content (when we restrict our attention to integer-based scales). But they differ with respect to interactiveness, and this explains the contrast between them as to whether they give rise to ignorance implicatures.

It is worth noting at this point that our analysis is also consistent with experimental evidence showing that comparative modifiers are mastered at an earlier developmental stage than their superlative counterparts (Musolino 2004, Geurts, Katsos, et al. 2010), and that superlative modifiers are harder to process than comparative modifiers (Geurts, Katsos, et al. 2010). Interactive propositions are arguably more complex than informative ones, and this complexity could lie behind those results. 
Raising and resolving issues with scalar modifiers

\subsubsection{Scalar and exhaustivity implicatures}

Recall from the introduction that three gives rise to scalar implicatures while at least three does not. This fact is reinforced by the following contrast:

(74) a. A: Three boys left.

B: No, four.

b. A: At least three boys left.

B: \#No, four.

A statement of the form $n$ Ps $Q$ can be denied on the grounds there are more than $n P$ s who $Q$, whereas this does not hold for statements of the form at least $n P$ s $Q$. The contrast also manifests itself in the fact that a perhaps rider is redundant with at least but not with a bare numeral:

(75) a. Three boys left, perhaps even four.

b. \#At least three boys left, perhaps even four.

This contrast between numerals with superlative modifiers and bare numerals is puzzling under the assumption that numerals have only a lower bound as part of their semantic content, and the upper bound arises through a scalar implicature (Horn 1972).

If we use a two-sided analysis of numerals, then the puzzle disappears; the 'no more than' inference is part of the conventional content of a bare numeral, and not part of the conventional content of a sentence with at least. But this case is an instance of a more general problem; there are examples not involving numerals in which $p$ gives rise to a quantity implicature but at least $p$ does not. In response to the question 'Who came to the party?', the answer 'Ann' implies that nobody else came to the party whereas 'at least Ann' does not imply that.

(76) Q: Who came to the party?
a. A: Ann.
$m$ Nobody else did.
b. $\mathrm{A}^{\prime}$ : At least Ann.
thr Nobody else did.

As we showed above, the two responses have the same informative content, but one is attentive and the other is not. This difference can be exploited to explain the contrast with respect to their scalar implicatures. 


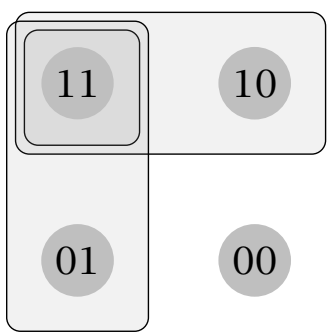

Figure 3 Question Under Discussion: 'Who snores?'

To do this, we build on the analysis of scalar implicatures laid out by Balogh (2009), who defines an exhaustification procedure giving the strengthened meaning of an inquisitive proposition in the context of a question under discussion. The basic idea is that exhaustification of a proposition $P$ in the context of a question under discussion $Q$ (also an inquisitive-style proposition) is the result of removing worlds $w$ from each of the possibilities $p$ in $P$ whenever $w$ is contained in one of the possibilities in $Q$ that is not implied by $p$. We formalize this as follows: ${ }^{6}$

\section{(77) Exhaustification}

$\operatorname{EXH}(P, Q)=\left\{p-q \mid p \in P\right.$ and $q=\left\{w \mid \exists q^{\prime} \in Q\left[w \in q^{\prime}\right.\right.$ and $\left.\left.\left.p \nsubseteq q^{\prime}\right]\right\}\right\}$

In our example, the question is 'Who snores?', and the possible answers are: 'Ann', 'Bill', and 'Ann and Bill'. The question is depicted in Figure 3. Applied to 'Ann snores' in the context of the question 'Who snores?', this yields the result that nobody else snores. From the single possibility in the denotation of 'Ann snores', namely $\left\{w_{10}, w_{11}\right\}$, we eliminate the world $w_{11}$, because that world is part of a non-implied alternative, 'Bill snores'. The result is $\left\{\left\{w_{10}\right\}\right\}$, as shown in Figure $4(\mathrm{a})$.

Now consider the case of 'At least Ann snores'. We have two possibilities to start with, $a=\left\{w_{10}, w_{11}\right\}$ and $a \& b=\left\{w_{11}\right\}$. Exhaustification causes $w_{11}$ to be removed from the first possibility, as before. But nothing is removed from the second possibility, because every alternative is either disjoint from it or implied by it, since it contains only one world. The result is $\left\{\left\{w_{10}\right\},\left\{w_{11}\right\}\right\}$, as depicted in Figure 4(b). The informational content of the exhaustified proposition allows for the possibility that Bill snores. Hence exhaustification does not result in the inference that Bill does not snore in this case.

6 We believe that this definition is equivalent to Balogh's; the only intended difference is that it does not involve 'possible propositions' as a subsidiary notion. 
Raising and resolving issues with scalar modifiers

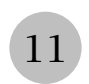

01
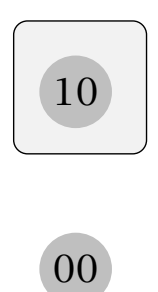

(a) $\operatorname{EXH}($ 'Ann')

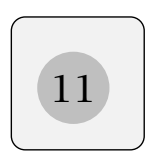

\section{0}

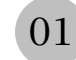

(b) $\mathrm{EXH}$ ('at least Ann')

Figure 4 Exhaustification of two propositions with respect to 'Who snores?'

Let us recap. We have shown that by analyzing superlative modifiers in inquisitive semantics, we can account for the fact that they do give rise to ignorance implicatures (unlike comparative modifiers), and that they do not give rise to scalar implicatures (unlike bare numerals). In the next two sections, we will show how the inquisitive analysis sheds light on how superlative modifiers interact with modals.

\subsection{Authoritative readings}

As Büring (2008) points out, the combination of at least with a universal modal gives rise to two different readings, the authoritative reading, which is the most prominent reading of (78), and the speaker insecurity reading, most prominent in (79).

(78) The paper must be at least 10 pages long.

(79) To become a member of this club, you have to pay at least $\$ 200,000$.

On its most prominent reading, (78) is false if it turns out that the paper has to be 15 pages long, while (79) allows for a higher amount of money to be the minimal requirement to be accepted in the club and would not be judged to be false if this is the case. The following sentence has both readings:

(80) John has to read at least three books.

a. Authoritative reading: The speaker knows what is allowed and what is not allowed, and in every acceptable world, there are three or more books that John reads. Furthermore, 
reading exactly three books is allowed, as is reading exactly four, etc.

b. Speaker insecurity reading: There is some number $n>3$ such that in every acceptable world, John reads $n$ books. The speaker does not know what is allowed and what is not allowed. On this reading, it does not follow that reading exactly three books is allowed, but the speaker considers it epistemically possible that reading three books is required.

Both readings are available under the present analysis.

The denotation under the speaker insecurity reading is a proposition with multiple possibilities, one for each element of the scale: John must read three books, John must read four books, etc. Let $p_{n}$ stand for the proposition 'John reads $n$ books'. Let us assume that must denotes a singleton set containing a function that takes a proposition and yields the proposition that the input proposition holds in all deontically accessible worlds:

$$
\llbracket m u s t \rrbracket=\{\lambda p . \square p\}
$$

We may assume that at least takes wide scope with respect to the modal. This gives the following result:

$$
\begin{aligned}
& \left\{\square p_{n} \mid n \geq 3\right\} \\
& =\left\{\square p_{3}, \square p_{4}, \square p_{5}, \ldots\right\}
\end{aligned}
$$

The Maxim of Interactive Sincerity gives us an ignorance implicature, to the effect that each of these requirements is epistemically accessible to the speaker. It does not follow that reading three books is allowed.

For the authoritative reading, we assume that the modal takes wide scope with respect to at least, and that Kratzer \& Shimoyama's (2002) operation of Existential Closure applies within the scope of the modal to gather all of the disjuncts into one possibility. Existential Closure over a set of possibilities $\left\{p_{1}, \ldots, p_{n}\right\}$ gives the singleton set consisting of the union of those possibilities $\left\{\bigcup\left\{p_{1}, \ldots, p_{n}\right\}\right\}$. (Following Kratzer and Shimoyama, we assume that this process is triggered inside the scope of a modal.) So under the authoritative reading, the denotation is a singleton set consisting of the proposition that is true of a world $w$ if and only if three or more books are read in every world accessible from $w$.

$\left\{\square \bigcup\left\{p_{3}, p_{4}, p_{5}, \ldots\right\}\right\}$ 
Raising and resolving issues with scalar modifiers

For the authoritative reading, we also want to derive what we may describe as a "distribution requirement" following Kratzer \& Shimoyama (2002), i.e. that all of the alternatives correspond to allowable options. As Alonso-Ovalle (2005) briefly points out, disjunctions give rise to distribution requirements under deontic necessity modals; here is an example:

(84) Dad, to Sandy: You must either clean your room or mow the lawn.

This sentence implies that it is permitted that Sandy cleans her room and it is permitted that Sandy mows the lawn. The kind of reasoning that explains the distribution requirement is spelled out by Kratzer \& Shimoyama (2002) as follows:

He picked the widest set of alternatives, $\{A, B\}$. Why didn't he pick $\{A\}$, which would have led to a stronger claim? It might be that $\square A$ is false. Or else, it might be that $\square A$ is true, but its exhaustivity inference $\neg \square B$ is false. We infer $\square A \rightarrow \square B$. The same kind of reasoning can be given for why she didn't pick $\{B\}$ and we infer $\square B \rightarrow \square A$.

Putting $\square[A \vee B]$ together with $\square A \leftrightarrow \square B$ makes it possible to infer both $\diamond A$ and $\diamond B$. Given that at least sentences denote sets of alternatives, just like disjunctions, analogous reasoning can be used to derive the distribution requirement for at least in the scope of a modal. ${ }^{7}$

The ability to account for the authoritative reading gives the present analysis an advantage over that of Geurts \& Nouwen (2007). As Büring (2008)

7 The example under consideration introduces some complications stemming from the fact that the alternatives are not logically independent as they are in (84). If we choose a twosided ("exactly") analysis of numerals, then the alternatives are mutually exclusive, and if we choose a one-sided ("n or more") analysis, there is a strict entailment relation among them. These situations change the reasoning by which the distribution requirement can be derived, to some extent. In neither case is there a potential exhaustivity inference to be avoided, and on the one-sided reading, the alternatives to be considered are not stronger. This is a general issue for the interpretation of alternative-introducing expressions in modal contexts, arising not just with superlative modifiers; You must read three or four books, for example, has a distribution requirement to the effect that exactly three books and exactly four books are both acceptable. Since three books is not stronger than three or four books on a one-sided analysis, Quantity does not straightforwardly dictate that a speaker choose the former when possible, so the hearer must have some other reason to wonder why that alternative wasn't chosen. It may be a preference for more homogeneous sets of alternatives in Groenendijk \& Roelofsen's (2009) sense. 
points out, "to derive the authoritative reading, [Geurts and Nouwen] have to assume an additional rule of 'modal concord' which can optionally turn the epistemic modals contributed by at least into deontic ones and subsequently delete the original deontic modal(s) corresponding to the matrix predicate." This rule of modal concord is problematic, as we discuss in $\S 4$. Like that of Büring (2008), the present account derives both readings through scope ambiguity.

Deriving the readings through scope ambiguity as above correctly predicts the speaker insecurity reading to be unavailable if at least cannot be raised out of the scope of the modal, as Büring points out. Consider the following example from Büring (2008), where at least $3 \%$ is inside a finite clause, blocking it from taking scope over the modal in the matrix clause:

(85) It was required that we pay at least 3\% to the agent.

This sentence cannot be used to express the insecurity of the speaker as to how much he has to pay his agent. This suggests that the scope ambiguity approach is on the right track.

\subsection{Missing readings puzzle}

Using inquisitive semantics also sheds light on the missing readings puzzle. Recall that (86a) cannot be used for the primary purpose of granting permission to have fewer than three beers, while (86b) can be.

(86) a. You may have at most two beers.

b. You may have fewer than three beers.

Hackl (2000) has already shown how to derive the two readings of (86b). According to Hackl, numbers like three denote individuals of type $d$ 'degree', and combine with a silent -many, defined as in (87), to produce a generalized quantifier. Here is an intensionalized version of Hackl's -many.

$$
\begin{aligned}
& \llbracket-m a n y \rrbracket=\lambda d \cdot \lambda P_{\langle e, p\rangle} \cdot \lambda Q_{\langle e, p\rangle} \cdot \lambda w \cdot \exists x[P(x)(w) \wedge Q(x)(w) \wedge \#(x)=d] \\
& \llbracket 3-m a n y \rrbracket=\lambda P_{\langle e, p\rangle} \cdot \lambda Q_{\langle e, p\rangle} \cdot \lambda w . \exists x[P(x)(w) \wedge Q(x)(w) \wedge \#(x)=3]
\end{aligned}
$$

Fewer than modifies three, and fewer than three denotes a quantifier over degrees: 
Raising and resolving issues with scalar modifiers

(89) $\llbracket$ fewer than three $\rrbracket=\lambda M_{\langle d, p\rangle} . \lambda w . \max \{n \mid M(n)(w)\}<3$

This quantifier cannot be interpreted in situ, so it undergoes $\mathrm{QR}$, and its trace is what combines with -many. The LF for the "pure permission" reading of (86b) is shown in (9oa) (ignoring the fact that the object quantifier also has to raise), and the truth conditions are expressed in stilted English in (9ob).

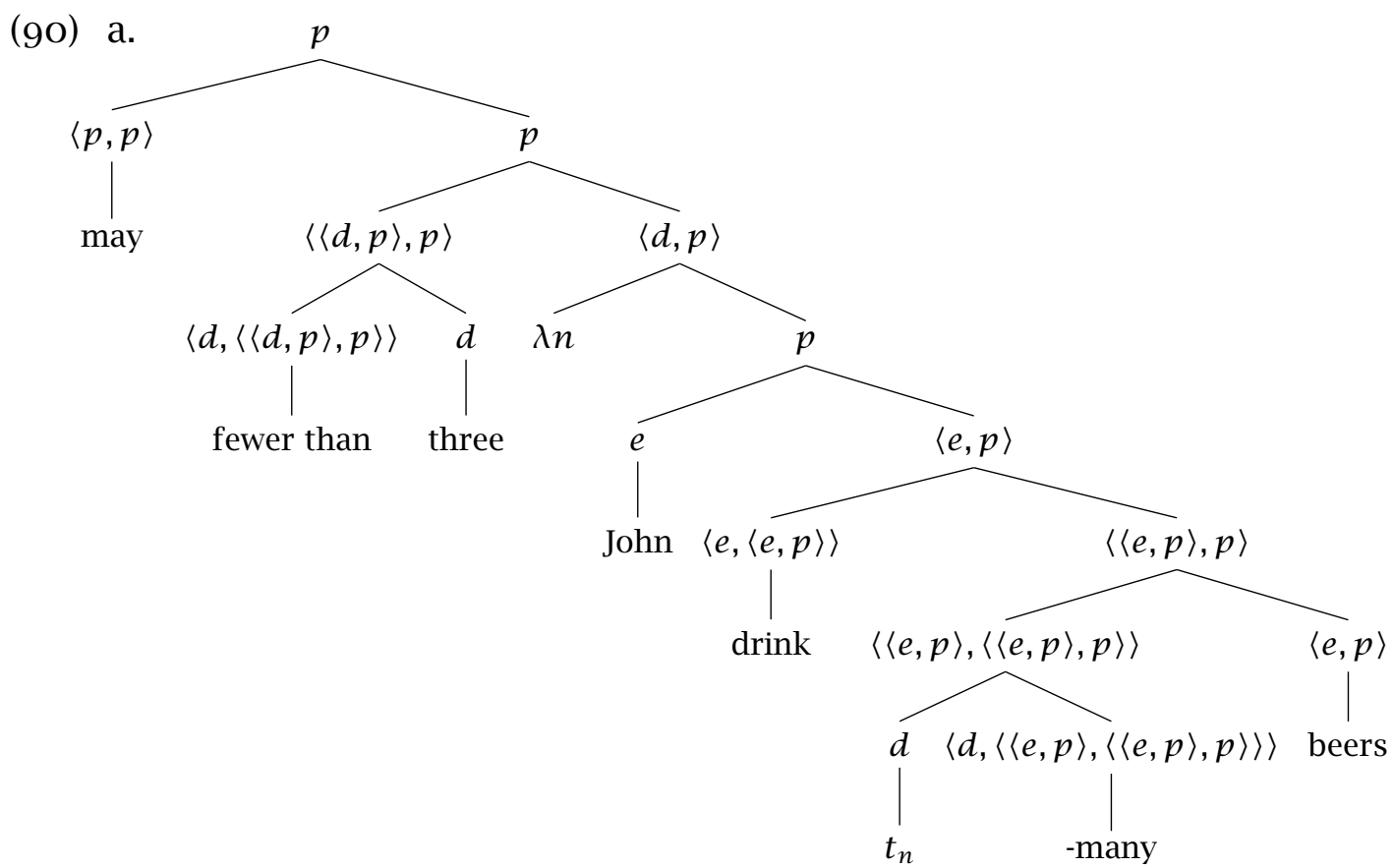

b. 'It is allowed that the greatest number of beers that John drinks is less than three.'

This is the "pure permission" reading because it expresses that it is acceptable to drink fewer beers, without ruling out having more. On the other reading, the permission modal takes scope inside fewer than three, giving the truth conditions: 'The greatest number $n$ such that it is allowed that there are $n$ beers that John drinks is less than three.' Hackl's assumptions about fewer than are quite compatible with everything we have said (although everything should be type-lifted into a singleton set consisting of its non-inquisitive meaning for the purposes of working in inquisitive semantics).

Our job here is to show that (86a) lacks the "pure permission" reading. While at most is not predicted to have the same semantic type as fewer than on our system (as it instantiates only modifier types of the form $\langle\tau, \tau\rangle$ ), it is possible to generate a reading on which the permission modal has wider 
scope than at most. The LF is shown in (91a) (with non-inquisitive semantic types), and the corresponding truth conditions are expressed in (91b). ${ }^{8}$

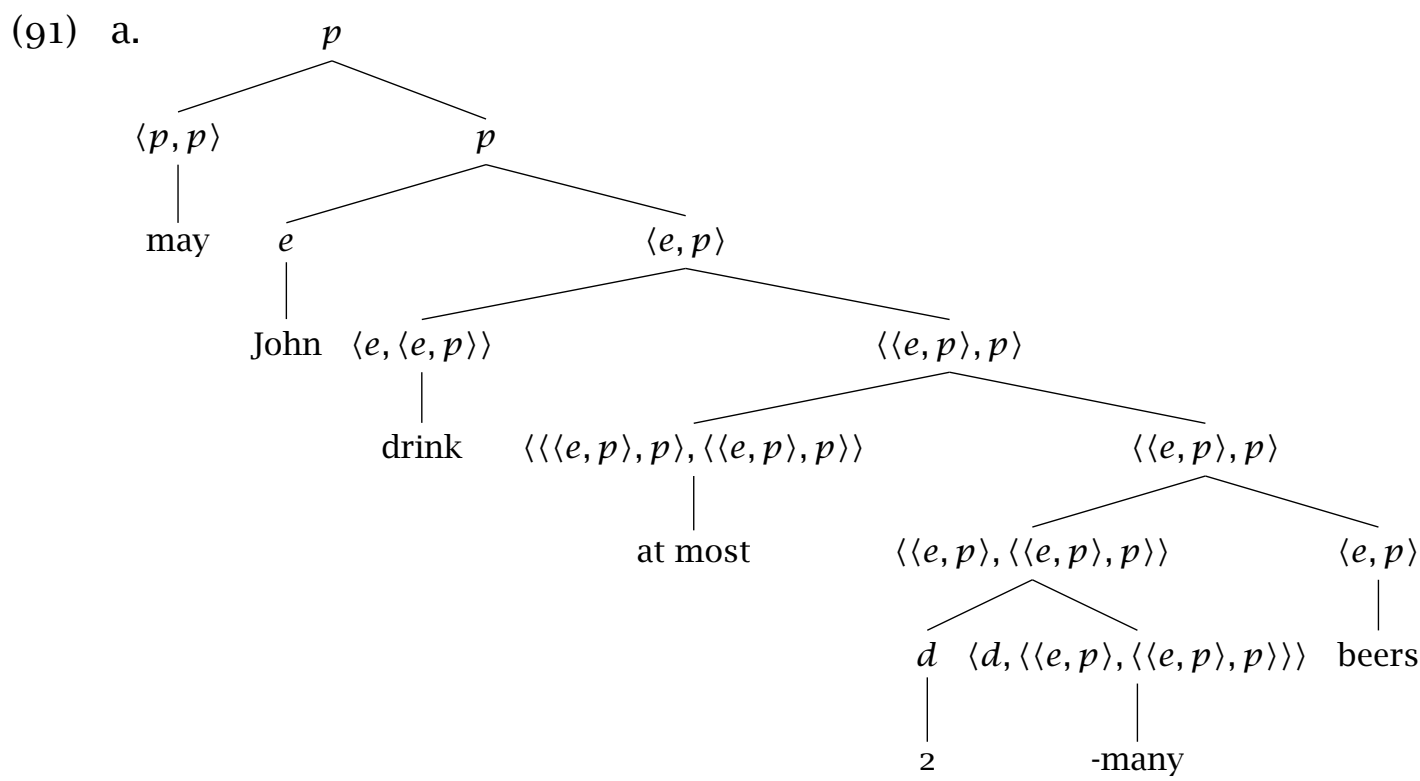

b. 'It is allowed that John drinks exactly o or 1 or 2 beers'

Here we seem to have generated the pure permission reading; these truth conditions are compatible with the possibility of drinking more beers. ${ }^{9}$

We propose that the possibility of drinking more beers is ruled out by implicature. Consider analogous cases of disjunction under permission modals. As Alonso-Ovalle (2005) points out, (92) implies that having crème caramel is not a permitted option.

(92) Mom, to Sandy: "You may have either this piece of cake or that ice cream."

8 We have to amend the definition of -many in order to make "John drinks o beers" one of the disjuncts. Assuming that objects cannot have null numerosity, the maximum $n$ such such that there is an $x$ such that $x$ is beers and John drank $x$ and $x$ has numerosity $n$ can never be o. We may assume that the relevant quantity is o in case there is no such $x$.

9 The alternatives at at most quantifies over are collected into a single possibility by Existential Closure (triggered in the scope of the modal), so the denotation is a singleton set. This means that the denotation is not interactive, and the Maxim of Interactive Sincerity does not apply, so we have an "authoritative reading". If at most were to take wide scope, then we would have a "speaker insecurity reading”, which could be glossed, 'John may drink exactly one beer or John may drink exactly 2 beers'. 
Raising and resolving issues with scalar modifiers

Alonso-Ovalle labels this the "exhaustivity requirement". Intuitively, the exhaustivity requirement seems like a quantity implicature: If crème caramel were a permitted option, then Mom would have included that one. The situation here is a bit different from the usual case of a quantity implicature though, because the statement would be truth-conditionally weaker if Mom had included a third option, since $\diamond[p \vee q \vee r]$ is weaker than $\diamond[p \vee q]$. However, if we consider the meaning strengthened with the distribution requirement, according to which all of the disjuncts correspond to allowable options, then the meaning that is ultimately communicated would have been stronger if Mom had included crème caramel as an option. The distribution requirement tells us that all of the disjuncts are allowable options. By adding a third disjunct, we would learn new information: that crème caramel is also allowed. The variant with three disjuncts is stronger in the sense that it conveys this additional information. Failure to include a third disjunct suggests that the additional information that would be conveyed is false, given that the speaker is an authority in this situation.

According to the inquisitive analysis of superlative modifiers that we are advocating, superlative modifiers are very much like disjunctions. We may therefore assume that analogous reasoning applies to (86a). Like disjunctions, superlative modifiers introduce a set of alternatives, and this set of alternatives is taken to be exhaustive in the same way. This leads to the implicature that for all $n$ greater than two, 'You may have $n$ beers' is false. Hence the intuition that (86a) conveys a prohibition.

This result depends on the assumption that focus is on the numeral, and the alternatives are all of the form 'You (may) have $n$ beers', with different values for $n$. The QUD has to be a 'how many?' question, in other words. The reading of ( $86 \mathrm{~b})$ that is not available for at most relies on a different kind of QUD, one that has only a binary partition over the set of possible worlds: 'Is it or is it not the case that the addressee may have fewer than three beers?' This reading requires verum focus. Why isn't this kind of QUD possible with (86a)? We suggest that it is because superlative modifiers are focus-sensitive operators like only, and like only, they require that focus be realized in the syntactic constituent to which they are a sister (Rooth 1985, 1992). This forces focus to fall on the numeral. (A yes/no question QUD would also be odd in combination with at most because neither of the answers would be stronger than the other, so at most would not be used to rule out stronger answers.) Combining this with the assumption that superlative modifiers introduce alternatives, we derive the result that a "pure permission" reading 
is not available for (86a), and that it conveys a limit on the range of allowable alternatives.

\subsection{Backwards compatibility check}

Now that we have an explanation for implicatures and the readings under modals, let us make sure that we have not lost what we gained in $\S 2$. The truth conditions are the same; they are just a bit more textured than they were before. The range of semantic types that superlative modifiers can combine with is still the same, and both entailment and non-entailment scales are still allowed.

The issue of focus is a bit more complex. We captured the focus-sensitivity of at least using the Focus Principle, which constrains the relationship between focus and the QUD ("Some part of a declarative utterance must evoke all of the possibilities in the QUD"). What it means to "evoke" possibilities was spelled out in terms of Rooth's alternative semantics for focus in Beaver \& Clark 2008; in an inquisitive framework, however, we must define this notion differently. To do so, we build on the analysis of focus in Balogh 20og. The focus principle is stated in terms of the notions of 'theme' and 'compliance'.

(93) Focus Principle (Balogh 2009: p. 53)

The theme of a focussed utterance must be compliant to the actual common ground.

The theme of an expression is essentially the result of existential closure over all focussed constituents. Balogh's framework is not compatible with the Montagovian framework we are working in here; she assumes translation of natural language expressions into a logical language, whereas we assume direct interpretation of natural language expressions. Therefore we cannot import Balogh's theory directly, but we assume an analogous theory that works on natural language expressions, providing existential closure over all focussed constituents. For example, the theme of Mary $y_{F}$ snores is Someone snores.

We may adopt the following definition of compliance, based on Groenendijk \& Roelofsen 2009.

\section{(94) Compliance}

A proposition $P$ (and any expression that denotes it) is compliant to $Q$ iff 
Raising and resolving issues with scalar modifiers

(a) every possibility in $P$ is the union of a set of possibilities in $Q$

(b) every possibility in the restriction of $Q$ by the informational content

in $P$ is included in a possibility in $P$

Part (a) ensures that $P$ is a partial or complete answer in Groenendijk \& Stokhof's (1984) sense. It rules out answers that are overly informative, e.g. "Anna and the moon is made of green cheese" in response to "Who snores?". It does not rule out answers that give no information, however, or answers that change the question under discussion. Part (b) requires that any change to the question under discussion constitutes progress towards answering the first question, by instituting a question that is easier to answer.

Now let us consider the interaction between focus and at least. Recall (26), repeated here.

(95) a. John should at least invite the [postdoc $]_{F}$ to lunch.

b. John should at least invite the postdoc [to lunch $]_{F}$.

The theme of (95a) is "John should invite someone to lunch" and the theme of (95b) is "John should invite the postdoc to something." These are compliant to different questions, and therefore the alternatives that at least quantifies over will be different in the two cases. This yields a difference in truth conditions between (95a) and (95b), because the ranking is not one of entailment. Thus, given a Balogh-style focus principle, we can account for the focus-sensitivity of at least even in our inquisitive setting.

\section{Comparison with previous work}

\subsection{QUD-based analyses}

As explained above, the analysis we sketched in \$2 is equivalent in a certain sense to Büring's (2008) analysis. Thus Büring's account fares empirically just as well as the non-inquisitive theory in §2. But we have argued that our inquisitive theory accounts more satisfactorily for the ignorance implicatures. Büring states that on his account, at least 'amounts to a disjunction', and provides an implicature schema that works on disjunctions, given in (38). But as pointed out in §3.1, the only level at which at least sentences are disjunctive on his theory is in the meta-language. The principles regulating implicatures cannot be sensitive to the syntax of the meta-language description of the meanings of lexical items. (Cummins \& Katsos (2010) and Biezma (2013) 
argue for a disjunctive approach similar to Büring's (2008), so this critique applies there as well.) Büring's idea that sentences with superlative modifiers are somehow disjunctive could potentially be implemented by invoking a syntactic transformation to LF and using an implicature schema that is sensitive to the syntax of the LF representation, or by using a translation to a formal language and using an implicature schema that is sensitive to the syntax of the formal language. But Büring did not advocate either of these solutions, and we prefer our own solution to both of them. With inquisitive semantics, it is not necessary to assume that there is any syntactic affinity between sentences with at least and disjunctions in order to give a unified account of their ignorance implicatures. Rather, under this view, the crucial feature that they share is at a deeper level. Under our theory, at least does not actually express a disjunction, but it shares a crucial feature with disjunctions: interactivity.

Using inquisitive semantics simultaneously solves the problem with scalar implicatures as well. Büring does not address the scalar implicature as far as we can see, but he may implicitly adopt Krifka's (1999) solution. Krifka (1999) accounts for the fact that superlative modifiers do not give rise to quantity implicatures by stipulating that at least has no alternatives. We see our proposal as less stipulative, because the assumption that superlative modifiers are interactive simultaneously accounts for the absence of quantity implicatures, the presence of ignorance implicatures, and the missing readings puzzle.

Furthermore, the inquisitive account sheds light on the difference between 'authoritative readings' and 'speaker insecurity readings'. Like Büring's account, and in contrast to Geurts \& Nouwen's (2007), our inquisitive account allows the modal to scope either below or above the superlative modifier, and the latter option makes the authoritative reading available. But the inquisitive account furthermore explains what the so-called 'speaker insecurity' reading has to do with speaker insecurity. Because the denotation is interactive on that scoping, it is subject to the Maxim of Interactive Sincerity, which generates an implicature of speaker insecurity. On Büring's account, it is not clear where speaker insecurity comes from on that scoping.

\subsection{Modal analysis: Geurts \& Nouwen (2007)}

Geurts \& Nouwen (2007) treat speaker insecurity as part of the semantics of superlative modifiers. They give two lexical entries for at least, one that 
Raising and resolving issues with scalar modifiers

modifies propositions, and one that modifies modifiers:

- If $\alpha$ is of type $t$, then $\llbracket$ at least $\alpha \rrbracket=\square \alpha \wedge \exists \beta[\beta \triangleright \alpha \wedge \diamond \beta]$

- If $\alpha$ is of type $\langle a, t\rangle$, then $\llbracket$ at least $\alpha \rrbracket=\lambda X[\square \alpha(X) \wedge \exists \beta[\beta \triangleright \alpha \wedge$ $\diamond \beta(X)]$ ]

Here, $\triangleright$ indicates pragmatic strength, so this analysis is similar to ours and Büring's insofar as pragmatic strength is involved.

This analysis is quite flexible, and allows at least to be applied to both numeral scales and adjectival scales. For example:

(96) Fred had at least three beers.

$$
\begin{aligned}
\square \exists x[\#(x)=3 \wedge \operatorname{BEER}(x) \wedge & \operatorname{HAVE}(\mathrm{F}, x)] \wedge \\
& \diamond \exists x[\#(x)>3 \wedge \operatorname{BEER}(x) \wedge \operatorname{HAVE}(\mathrm{F}, x)]
\end{aligned}
$$

(97) This is at least warm.

$$
\square \operatorname{WARM}(\mathrm{s}) \wedge \diamond[\operatorname{HOT}(\mathrm{s}) \vee \operatorname{SCALDING}(\mathrm{s})]
$$

The presence of an epistemic possibility modal explains the fact that sentences with at least and at most convey ignorance on the part of the speaker. Comparative modifiers do not signal epistemic possibility on this theory, and this explains the contrast between superlative and comparative modifiers with respect to ignorance.

As argued by Cohen \& Krifka (2011), however, this approach crucially forfeits extensionality. Treating superlatives as epistemic operators makes them dependent on the beliefs of the speaker. Thus if rabbits and magical beings are coextensional and the speaker does not know this, then "Mary petted at least three rabbits" is falsely predicted not to be equivalent to "Mary petted at least three magical beings."

Some of the experimental results obtained by Cummins \& Katsos (2010) also show that the truth conditions are not quite right. Recall (32), repeated here as (98).

(98) a. If Berta has had at most three drinks, she is fit to drive. Berta has had at most two drinks.

b. Berta is fit to drive.

As Cummins \& Katsos (2010) discuss, this inference should not be valid according to Geurts and Nouwen, but native speakers consistently judge it as valid. 
Another problem for Geurts and Nouwen's theory is that they must appeal to a non-compositional and somewhat mysterious mechanism of 'modal concord' in order to explain authoritative readings. Recall (78), repeated here:

(99) The paper must be at least 10 pages long.

Büring (2008)

This sentence has a reading where the deontic modal scopes over the at least phrase, and this is what Büring (2008) refers to as the 'authoritative reading'. If the epistemic possibility modal that is supposed to be introduced by at least scopes underneath the universal deontic modal, the result is:

(100) In all deontically accessible worlds, it is epistemically necessary that the paper has (at least) 10 pages and epistemically possible that the paper has more than 10 pages.

The opposite scoping (at least > must) would give:

(101) It is epistemically necessary that in all deontically accessible worlds, the paper has (at least) 1o pages, and it is epistemically possible that in all deontically accessible worlds, the paper has more than 10 pages.

The latter (labelled (101)) is the speaker insecurity reading. The former (labelled (100)) is not the authoritative reading.

Geurts and Nouwen invoke the notion of modal concord to account for this case, where the universal modal of at least gets fused with the universal deontic modal introduced by must. They stipulate that the 'primary operator' of at least is the necessity operator, and that the 'primary operator' of at most is the possibility operator. Modal concord results in a fusion of the primary operator with that of a higher modal, so universal modals can fuse with universal modals and existential modals can fuse with existential modals. The modal flavor is taken from the higher modal. (As Nouwen (2010) points out, this is a non-compositional process.) In the case of (99), this means that the epistemic necessity operator of at least gets fused with the deontic necessity operator introduced by must, taking on its deontic flavor. The result is the authoritative reading. They also stipulate that epistemic operators cannot outscope deontic ones, and thereby rule out the ordinary 'compositional' reading that their account would generate.

Geurts and Nouwen do not spell out exactly how modal concord is supposed to work, so it is difficult to know what kind of predictions it makes, but it would seem to be a Pandora's Box of interpretations for sentences 
Raising and resolving issues with scalar modifiers

that are never found. For example, suppose that Bill sees a man in a hurry carrying a laptop and a projector dongle and Bill says:

(102) He must have to give a presentation soon.

By this Bill would mean that it is epistemically necessary that it is deontically necessary for him to give a presentation. Modal concord would apply here according to the constraints suggested by Geurts and Nouwen because the two modals are both universal modals, and would give the reading: 'it is epistemically necessary that he is giving a presentation', which is not what the sentence means. Similarly, recall (85) from Büring (2008), repeated here:

(103) It was required that we pay at least 3\% to the agent.

This should have a modal concord reading, but it does not. The scope-based account, on the other hand, correctly predicts that such a reading is not available in this case.

We also find the notion that modal concord only targets one of the conjuncts in the superlative modifier problematic. Geurts and Nouwen say that the conjuncts of the meaning of at least and at most have a "different status", where one "seems less central", and it is the one that is more "central" that is targeted by modal concord. They suggest that the second conjunct might be "pragmatically derived" from the first conjunct as an implicature, but this does not really work (especially for at most) as they discuss in their section 9. They also rule out an analysis of the second component as a conventional implicature, and the same argument applies to the idea that it is a presupposition, so it is very difficult to imagine what this supposed difference in status is supposed to amount to.

Finally, Geurts \& Nouwen's (2007) analysis also falsely predicts that John is at least an assistant professor implies that (it is neccessary according to the speaker that) John is an assistant professor. This is not what the sentence means. The sentence would be true and felicitous if John is a full professor, and a full professor is not an assistant professor. Geurts and Nouwen revise their theory in an appendix in order to fix this problem, so that at least $p$ means 'something at least as strong as $p$ is necessary and something stronger than $p$ is possible'. It is not clear that necessity is still the 'primary operator' after this revision, so it is not clear that this change maintains the virtues of the modal concord story. This revision also does not straightforwardly predict an ignorance implicature, because the proposition 
that is both necessary and possible according to the speaker might be the same one.

To summarize, the notion of modal concord is problematic because it is non-compositional, incompletely worked out, relies on a dubious distinction between "primary" and "non-primary" modal operators, overgenerates, and undergenerates. Geurts \& Nouwen's (2007) account also fails to capture the correct truth conditions, because it is not extensional, and runs into problems when it comes to non-entailment scales. All of these problems are avoided under the present account.

\subsection{Maxima/minima indicators: Nouwen (2010)}

Nouwen (2010) proposes that modified numerals can be divided into two separate classes, A and B, and superlatives are in class B. Class B modifiers have the following meaning, where $\mathrm{MOD}_{B}^{\downarrow}$ stands for upper bounded class B modifiers (at most, maximally, up to, ...) and $\mathrm{MOD}_{B}^{\dagger}$ for the lower bound modifiers (at least, from, minimally, ...).

(104) $\llbracket M O D_{B}^{\downarrow} \rrbracket=\lambda d \cdot \lambda M \cdot \max _{n}(M(n))=d$

(105) $\llbracket M O D_{B}^{\dagger} \rrbracket=\lambda d \cdot \lambda M \cdot \min _{n}(M(n))=d$

These combine with a numeral that has combined with one of two different possible silent -manys, one which introduces existence, and one which introduces both existence and uniqueness.

This proposal only deals with superlative modifiers in their function as modifiers of numerals, and therefore does not account for their wider distribution or focus-sensitivity. Furthermore, the account of the ignorance implicature with at most relies on an unspecified "reinterpretation" process introducing a speaker epistemic possibility modal. Nouwen's semantics generates a reading for (106) on which it is equivalent to (107) on an "exactly" reading.

(106) Jasper invited at most 10 people.

(107) Jasper invited 10 people.

Nouwen claims that this reading is blocked because it can be expressed through a simpler form. The hearer therefore reinterprets (106) to be a 
Raising and resolving issues with scalar modifiers

statement about what the speaker holds possible, and an epistemic possibility modal for the speaker is introduced into the interpretation. It is not clear how this reinterpretation process works or under what circumstances it applies. For example, as Nouwen notes in footnote 12, acknowledging an anonymous reviewer, the same reasoning should apply to exactly 10 ; this should get reinterpreted with some kind of speaker possibility modal since there is a simpler way of expressing the same idea, namely (107). On our account, the ignorance implicatures follow much more straightforwardly. ${ }^{10}$

\subsection{Speech act analysis: Cohen \& Krifka (2011)}

Cohen \& Krifka (2011) analyze superlative modifiers as illocutionary operators. According to this view, to express at least $\alpha$ is to constrain the possible future developments of the conversation. A conversation consists of a sequence of commitments on the part of the participants (the commitment development), and meta-speech acts serve to constrain future potential commitments. One such meta-speech act is GRANT (somewhat misleadingly named). To GRANT $\phi$ is to express the negation of the speech act of asserting $\neg \phi$. Negating a speech act ("denegation") is not quite like negating a proposition; for example, when one says I don't promise to come, one is negating the speech act of promising. Using $\sim$ as the symbol for denegation, $\operatorname{GRANT}(\phi)$ is defined as $\sim \operatorname{ASSERT}(\neg \phi)$. In other words, a speaker who GRANTS $\phi$ indicates that he or she is unwilling to assert $\neg \phi$. This rules out future developments of the conversation on which the speaker is committed to $\neg \phi$.

Superlative modifiers are quantifiers over GRANTs on this view. For example, at least three $\phi$ expresses that the speaker refuses to GRANT, for all $n$ less than three, that John petted $n$ rabbits. Cohen and Krifka represent the meaning of (108) as in (109).

(108) John petted at least three rabbits.

(109) $C+\bigwedge_{n<3} \sim \operatorname{GRANT}(|\operatorname{RABBIT}| \cap|\lambda x . \operatorname{PET}(\mathrm{J}, x)|=n)$, where $C$ is a commitment space

10 Schwarz, Buccola \& Hamilton (2012) offer a further critique of Nouwen (2010); they argue that Nouwen's Class B modifiers should not be lumped together so coarsely. We have not addressed any of the other 'Class B' modifiers in the present article, so their criticism does not apply to our proposal. 
Again, $\sim$ is the 'denegation' symbol, and $\bigwedge$ symbolizes a kind of generalized conjunction that applies to meta-speech acts, and + represents how the commitment space is updated. This formula says that the speaker does not GRANT that John petted zero rabbits, one rabbit, or two rabbits.

As this account operates on the speech act level, it is a non-trivial question what the very truth-conditions of sentences involving superlatives are. It seems clear, though, that since (108) disallows a development of the conversation on which the speaker becomes committed to John's petting of o, 1 or 2 rabbits, the sentence can be said to be false under those conditions. This is a desirable prediction.

To capture the conditions under which the sentence is true poses a bigger problem according to Cohen and Krifka. (108) should turn out to be true if John petted exactly four rabbits. Cohen and Krifka say that this circumstance would not be "sufficient to account for the truth of" (108), because the speaker "could, for example, also assert" John did not pet exactly four rabbits (p. 22). They say (p. 22), "In this case, it would still be the case that the minimal $n$ s.t. the speaker GRANTs that John petted exactly $n$ rabbits is three [so the requirements imposed by at least according to their theory would be satisfied], yet [(108)] would be false, rather than true." Here they are implying that if the speaker can consistently assert that John did not pet exactly four rabbits, then John petted at least three rabbits is false. But just because the sentence is true if John petted exactly four rabbits doesn't mean that the sentence is false if John didn't pet exactly four rabbits. Suppose John petted exactly five rabbits. Then a speaker could truthfully assert that John did not pet exactly four rabbits, and it is still perfectly true that John petted at least three rabbits. The sentence will also be true if John petted exactly four rabbits. Just because a speaker could consistently assert that something is not the case does not mean that the sentence is false when it is the case. So the possibility of consistently asserting John did not pet exactly four rabbits does not preclude (108) being true when John petted exactly four rabbits.

Nevertheless, to remedy this perceived problem, Cohen and Krifka assume that at least "does not have standard truth conditions: to get a proposition from it, we need a scalar implicature" (p. 32). We admit that we do not understand how this works. Here is how Cohen and Krifka explain it (p. 22):

By using a superlative quantifier, the speaker took the trouble to indicate that she accepts the commitments of all of the assertions in (6o) [namely: John did not pet exactly zero rabbits, 
Raising and resolving issues with scalar modifiers

John did not pet exactly one rabbit, and John did not pet exactly two rabbits]; if she also wanted to commit to the claim that John did not pet exactly $n$ rabbits for other values of $n$, the maxim of Quantity dictates that she should have indicated that as well. From the fact that she didn't, we can conclude, by a straightforward implicature, that she is not committed to such an assertion. Since the content of all the assertions that the speaker is committed to is true, it follows that [(108)] is true if John petted exactly four rabbits, which is the result we want.

We do not understand what "all the assertions that the speaker is committed to" refers to in this passage, nor how the truth of their content leads to the desired conclusion. If the assertions that the speaker is committed to (via scalar implicature) are those that the speaker is not committed to the negation of, then the speaker must be committed to an infinite number of contradictory assertions, including John petted exactly four rabbits and John petted exactly five rabbits. And we cannot even assume that the speaker is committed to even one of these; John petted at least three rabbits does not imply in any way, either through entailment or implicature, that John petted exactly four rabbits. So we cannot understand their explanation.

The notion that conversational implicature is required for "complete truth conditions" plays a crucial role in their explanation for the fact that at least does not always sound acceptable under negation, as Geurts \& Nouwen (2007) pointed out, e.g.:

(110) a. *None of the guests danced with at least/most three of the waitresses.

b. *Betty didn't have at least/most three martinis.

(111) a. John hardly ate \{??at least three / more than two $\}$ apples.

b. This won't take \{???at least 50 / more than 45$\}$ minutes.

Since negation is a downward-entailing environment, scalar implicatures do not arise there, so at least sentences lack complete truth conditions in such environments, according to Cohen and Krifka. As Cohen and Krifka put it on p. 32, "Since [the complete truth conditions of scalar modifiers] are generated by scalar implicature, it follows that it should not be possible to embed them in a downward entailing context." 
As the foregoing discussion implies, Cohen \& Krifka (2011) make a distinction between truth conditions and falsity conditions, stating that the falsity conditions of superlative modifiers follow semantically, while the truth conditions follow through a scalar implicature. We find this idea conceptually problematic, because it conflates the distinction between implicature and entailment entirely. Fundamental to the notion of implicature is that a sentence can be true without any of its implicatures being true. For example, "Some of the students passed" can be true even if not all of the students passed - indeed, this possibility is what shows that "Not all of the students passed" is an implicature rather than an entailment. Cohen and Krifka would presumably not want to say that the truth conditions of some are derived by scalar implicature, but let us consider what it would mean if they were. Suppose that the truth conditions for "Some of the students passed" were derived by scalar implicature. That would mean that in order for "Some of the students passed" to be true, "Not all of the students passed" would have to be true. But if that were the case, we would say that "Some of the students passed" entails "Not all of the students passed." 11 The same applies to superlative modifiers: If the truth of a statement containing a superlative modifier depends on some other statement being true, then the latter statement is entailed, not conversationally implicated. So, given a distinction between implicature and entailment, it seems conceptually impossible that truth conditions could be "derived via scalar implicature".

However, as they point out, the negation data is a problem for the type of account that Büring pursues involving disjunction, because disjunctions do not seem to behave quite in the same way as superlative modifiers under negation. (112a) is attested and sounds natural, but the corresponding example with at least sounds odd. With more than, the sentence sounds natural again.

(112) a. Nobody wants to spend three days or more in hospital if they could be safely back home within 24 hours.

b. \#Nobody wants to spend at least three days in hospital if they could be safely back home within 24 hours.

c. Nobody wants to spend more than two days in hospital if they could be safely back home within 24 hours.

11 Here is a standard definition of entailment (Chierchia \& McConnell-Ginet 1990): A entails B if and only if whenever $\mathrm{A}$ is true, $\mathrm{B}$ is true too. 
Raising and resolving issues with scalar modifiers

This suggests that we cannot use interactiveness to explain the embedding facts. And indeed, from a theoretical perspective, interactiveness does not predict that these elements should not be embeddable. ${ }^{12}$ Therefore this data remains unaccounted for under our account.

As Cohen and Krifka discuss, based on observations from Nilsen 2007, there are cases in which superlative modifiers can be embedded in downwardentailing environments, and interestingly, these seem limited to contexts in which pragmatic strength is aligned with some evaluative "goodness" (with some exceptions). Cohen and Krifka propose that there are two at leasts, one of which is specified for evaluative scales and capable of appearing under negation. Following the terminology of Nakanishi \& Rullmann (2009) we may call the at least that can convey speaker ignorance epistemic, and the evaluative one concessive.

We find the assumption of two separate lexical entries plausible based on how their properties cluster together, as the following examples from Nakanishi \& Rullmann (2009) shows. One difference between them is that the concessive entails the prejacent. For example, (113a) does not imply that Mary is an associate professor but (113b) does.

(113) a. Mary is at least an associate professor.

epistemic

b. At least Mary is an associate professor.

concessive

Furthermore, an epistemic interpretation is not possible when no higher alternatives are known to be true, while this is not the case for the concessive interpretation:

(114) a. \#Mary didn't win a gold medal, but she won at least a silver medal. epistemic

b. Mary didn't win a gold medal, but at least she won a silver medal. concessive

Finally, as hinted at by the term 'evaluative' used by Cohen and Krifka, the concessive indicates the prejacent is dispreferred (that one is "settling for less"), while epistemic at least does not convey such a judgment:

(115) a. Phelps won at least eight medals. epistemic

b. \#At least Phelps won eight medals. concessive

12 However, notice that disjunctions are sometimes bad in the same places as superlatives:

(i) ???John hardly ate three or more apples.

(ii) ???This won't take 45 or more minutes. 
As these examples show, the epistemic interpretation arises from sentencemedial uses of at least, while sentence-initial uses tend to give rise to the concessive interpretation. Biezma (2013) argues that the difference between these interpretations is not simply a matter of syntactic distribution, and that the context plays a crucial role in the selection of one over the other. Biezma aims to give a unified analysis of these two uses of at least. According to her analysis, for a concessive interpretation to arise it has to be known that no higher alternatives are true. Then no scalar implicature is triggered, and it follows that the prejacent is true. We are still not entirely convinced that there are not two separate lexical items; Biezma's account does not explain the evaluativity of concessive at least, nor the difference in syntactic distribution. So we side with Cohen and Krifka on this issue, assuming there to be two separate lexical items.

But contrary to what Cohen and Krifka predict, epistemic at least can occur in downward-entailing environments:

(116) a. Everyone who was at least an assistant professor was invited, and nobody else. $\quad(\Rightarrow$ Full professors were invited)

b. Everyone who reads at least 40 hours gets one entry to the "super raffle".

c. Attendance is FREE to everyone who brings at least one boat for others to try.

These uses of at least cannot be concessive: (116a) should only be possible under a concessive interpretation if it is known that nobody is a full professor, as the use of concessive at least presupposes that $x$ is not higher on the scale. Furthermore, (116b) and (116c) do not naturally give rise to a "settle for less"/"evaluative” reading, as the consequences of fulfilling the minimal requirement laid out by at least is arguably positive and not ranked low with respect to the expectations of the addressees. Therefore, while it remains an interesting puzzle why evaluative scales seem to be required in downwardentailing environments, we conclude that Cohen and Krifka's explanation based on the assumption that there is a separate lexical item does not seem to capture all the facts.

One might wonder whether it is possible to embed sentences with superlative modifiers at all under Cohen \& Krifka's (2011) account. The answer is yes, if the embedding operator can embed speech acts. To account for Mary thinks that John petted at least three rabbits, for example, they state 
Raising and resolving issues with scalar modifiers

that meta-speech acts are similar to propositional attitudes, "and hence are predicted to be embeddable under [propositional attitude verbs]" (p. 46). What about authoritative readings, as in The paper has to be at least 10 pages long? The modal has to be able to embed speech acts in order to take wide scope over at least. Cohen and Krifka discuss the example John needs at least three martinis, and they say that in order to get the wide scope reading, they "are treating the verb need as a sort of meta-speech act. Specifically, the verb must be able to subcategorize for speech acts, i.e. the subject must be an entity that is capable of GRANTing (typically a human)." In a case like The paper has to be at least 10 pages long, there is no human subject, so it does not appear as if their solution will carry over to this case.

To summarize, there appear to us to be some problems with Cohen and Krifka's account, although the predictions are not easy to evaluate. Crucially, we are not convinced by their explanation for the constraints on embedding of superlative modifiers under negation, and the system does not seem to generate superlative modifiers embedded under necessity modals, hence authoritative readings, assuming that necessity modals are not meta-speech

acts. Furthermore, the account relies on the assumption that truth conditions can be derived via scalar implicature, which we have argued to be conceptually problematic. Our account is comparatively mundane and provides a clear account of the ignorance implicature and authoritative readings, though we admit that there is still work to be done in order to explain the limited distribution of superlative modifiers under negation.

\section{Summary}

We have proposed an analysis of the superlative modifiers at least and at most that accounts for the relationship between them and only, when paired with the analysis of only in Beaver \& Clark 2008 and Coppock \& Beaver 2011. This analysis also successfully explains its truth conditions, its flexibility in distribution, its focus-sensitivity, and its ability to give rise to authoritative readings.

We have argued further that superlative modifiers are interactive, meaning that they raise issues to be resolved. Given the Maxim of Interactive Sincerity, this allows us to account for the fact that superlative modifiers give rise to ignorance implicatures. We have argued that comparative modifiers are not interactive, and hence they do not give rise to ignorance implicatures. Furthermore, using Balogh's (2009) exhaustivity operator, we can account 
for the fact that superlative modifiers do not give rise to scalar implicatures, while corresponding sentences lacking them do. The inquisitive analysis also sheds light on the interaction between superlative modifiers and modals.

One issue that remains unsolved is the contrast between superlative and comparative modifiers in certain negative environments observed by Cohen \& Krifka (2011). This point aside, however, the present analysis captures all of the facts mentioned in the introduction, unlike any previous analysis. The analysis is moreover relatively mundane, avoiding appeal to exotic notions like modal concord, reinterpretation, and truth conditions derived by implicature, and relatively general, making use of independently motivated pragmatic principles. One theoretical innovation, however, has been to bring pragmatic strength rankings into inquisitive semantics. It will be interesting to see what other interactions we may find between pragmatic strength and inquisitivity in future research.

\section{References}

Alonso-Ovalle, Luis. 2005. Distributing the disjuncts over the modal space. North East Linguistic Society (NELS) 35. http://people.linguistics.mcgill.ca/ luis.alonso-ovalle/papers/alonso-ovalle_NELS2005.pdf.

Balogh, Kata. 2009. Theme with variations: A context-based analysis of focus. University of Amsterdam PhD thesis. http://www.illc.uva.nl/Research/ Dissertations/DS-2009-07.text.pdf.

Barwise, Jon \& Robin Cooper. 1981. Generalized quantifiers and natural language. Linguistics and Philosophy 4(2). 159-219. http://dx.doi.org/10. 1007/BFo0350139.

Beaver, David \& Brady Z. Clark. 2008. Sense and sensitivity: How focus determines meaning. Chichester: Wiley-Blackwell. http://dx.doi.org/10.1002/ 9781444304176.

Biezma, María. 2013. Only one at least: Refining the role of discourse in building alternatives. In University of Pennsylvania working papers in linguistics, vol. 19. Penn Linguistics Club. http://repository.upenn.edu/ pwpl/vol19/iss1/3.

Büring, Daniel. 2008. The least at least can do. West Coast Conference on Formal Linguistics (WCCFL) 26. 114-130. http://www.lingref.com/cpp/ wccfl/26/paper1662.pdf.

Chierchia, Gennaro \& Sally McConnell-Ginet. 1990. Meaning and grammar: An introduction to semantics, 2nd. Cambridge, MA: MIT Press. 
Raising and resolving issues with scalar modifiers

Ciardelli, Ivano, Jeroen Groenendijk \& Floris Roelofsen. 20o9. Attention! Might in inquisitive semantics. Semantics and Linguistic Theory (SALT) 19. http://elanguage.net/journals/salt/article/view/19.6.

Ciardelli, Ivano, Jeroen Groenendijk \& Floris Roelofsen. 2012. Inquisitive semantics. NASSLLI 2012 lecture notes. https://sites.google.com/site/ inquisitivesemantics / documents/NASSLLI-2012-inquisitive-semanticslecture-notes.pdf.

Cohen, Ariel \& Manfred Krifka. 2011. Superlative quantifiers as modifiers of meta-speech acts. The Baltic International Yearbook of Cognition, Logic and Communication 6. http://dx.doi.org/10.4148/biyclc.v6io.1578.

Coppock, Elizabeth \& David Beaver. 2011. Sole sisters. Semantics and Linguistic Theory (SALT) 21. 197-217. http://elanguage.net/journals/salt/article/ view/21.197.

Coppock, Elizabeth \& David Beaver. 2012a. Exclusive updates. In Maria Aloni, Floris Roelofsen, Galit Weidman Sassoon, Vadim Kimmelman Katrin Schulz \& Matthijs Westera (eds.), 18th Amsterdam Colloquium, 291-30o. Berlin: Springer. http://dx.doi.org/10.1007/978-3-642-31482-7_30.

Coppock, Elizabeth \& David Beaver. 2012b. Mere-ology: Toward a unified analysis of mere and other exclusives. In Anamaria Falaus (ed.), Alternatives in semantics, 150-173. Palgrave.

Coppock, Elizabeth \& David Beaver. to appear. Principles of the exclusive muddle. Journal of Semantics.

Cummins, Chris \& Napoleon Katsos. 2010. Comparative and superlative quantifiers: Pragmatic effects of comparison type. Journal of Semantics 27(3). 271-305. http://dx.doi.org/10.1093/jos/ffqoo6.

Geurts, Bart, Napoleon Katsos, Chris Cummins, Jonas Moons \& Leo Noordman. 2010. Scalar quantifiers: Logic, acquisition, and processing. Language and Cognitive processes 25(1). 130-148. http://dx.doi.org/10.1080/ o1690960902955010.

Geurts, Bart \& Rick Nouwen. 2007. At least et al. The semantics of scalar modifiers. Language 83(3). 533-559. http://dx.doi.org/10.1353/lan.2007. O115.

Groenendijk, Jeroen \& Floris Roelofsen. 2009. Inquisitive semantics and pragmatics. Presented at the Workshop on Language, Communication, and Rational Agency at Stanford, May 2009. http://www.illc.uva.nl/ inquisitivesemantics. 
Groenendijk, Jeroen \& Martin Stokhof. 1984. Studies on the semantics of questions and the pragmatics of answers. University of Amsterdam $\mathrm{PhD}$ thesis. http://dare.uva.nl/en/record/123669.

Hackl, Martin. 2000. Comparative quantifiers. Cambridge, MA: Massachusetts Institute of Technology PhD thesis. http://dx.doi.org/1721.1/8765.

Hamblin, C. L. 1973. Questions in Montague English. Foundations of Language 10. 41-53. http://www.jstor.org/stable/25000703.

Horn, Laurence R. 1969. A presuppositional analysis of only and even. Chicago Linguistic Society (CLS) 5. 98-107.

Horn, Laurence R. 1972. On the semantic properties of logical operator in English. Los Angeles, CA: University of California PhD thesis.

Horn, Laurence R. 1992. The said and the unsaid. In Chris Barker \& David Dowty (eds.), Semantics and linguistic theory (SALT), vol. 2, 163-192. Columbus: OSU Working Papers in Linguistics.

Jacobson, Pauline. 1999. Towards a variable-free semantics. Linguistics and Philosophy 22(2). 117-185. http://dx.doi.org/10.1023/A:1005464228727.

Kaplan, David. 1999. The meaning of ouch and oops. Lecture presented at the University of California at Berkeley.

Kennedy, Chris. 2012. A scalar semantics for scalar readings of number words. Manuscript, University of Chicago. http://semanticsarchive.net/Archive/ zIyMjg1Y/numberwords-ck.pdf.

Kratzer, Angelika \& Junko Shimoyama. 2002. Indeterminate pronouns: The view from Japanese. In Yukio Otsu (ed.), Proceedings of the third Tokyo conference on psycholinguistics, 1-25. Tokyo: Hituzi Syobo. http://semanticsarchive. net/Archive/WEwNjc4Z/.

Krifka, Manfred. 1999. At least some determiners aren't determiners. In Ken Turner (ed.), The semantics/pragmatics interface from different points of view, 257-291. Oxford: Elsevier. http://amor.cms.hu-berlin.de/ h2816i3X/ Publications/ATLEASTSOME.pdf.

Montague, Richard. 1974a. English as a formal language. In Richmond H. Thomason (ed.), Formal philosophy, 188-221. New Haven: Yale University Press.

Montague, Richard. 1974b. The proper treatment of quantification in ordinary English. In Richmond H. Thomason (ed.), Formal philosophy. Yale University Press.

Musolino, Julien. 2004. The semantics and acquisition of number words: integrating linguistic and developmental perspectives. Cognition 93(1). 1-41. http://dx.doi.org/10.1016/j.cognition.2003.10.002. 
Raising and resolving issues with scalar modifiers

Nakanishi, Kimiko \& Hotze Rullmann. 2009. Epistemic and concessive interpretation of at least. Slides for a paper presented at the meeting of the Canadian Linguistics Association, Carleton University. http://www. linguistics.ubc.ca/sites/default/files/2009.Nakanishi_Rullmann.CLA_ .pdf.

Nilsen, Oystein. 2007. At Least: Free choice and lowest utility. Talk given at the ESSLLI Workshop on Quantifier Modification, Dublin. http://www. hum.uu.nl/medewerkers/o.nilsen/atleastEssllibeamer.pdf.

Nouwen, Rick. 2010. Two kinds of modified numerals. Semantics and Pragmatics 3(3). 1-41. http://dx.doi.org/10.3765/sp.3.3.

Pruitt, Kathryn \& Floris Roelofsen. 2011. Disjunctive questions: Prosody, syntax, and semantics. Presented at a seminar at the Georg August Universität Göttingen, April 2011.

Roberts, Craige. 1996/2012. Information structure in discourse: Towards an integrated formal theory of pragmatics. Semantics and Pragmatics 5(6). 1-69. http://dx.doi.org/10.3765/sp.5.6.

Roelofsen, Floris \& Sam van Gool. 2010. Disjunctive questions, intonation, and highlighting. In Maria Aloni, Harald Bastiaanse, Tikitu de Jager \& Katrin Schulz (eds.), Logic, language and meaning: Selected papers from the 17th Amsterdam Colloquium, 384-394. Berlin: Springer. http://dx.doi. org/10.1007/978-3-642-14287-1_39.

Rooth, Mats. 1985. Association with focus. Amherst, MA: University of Massachusetts $\mathrm{PhD}$ thesis.

Rooth, Mats. 1992. A theory of focus interpretation. Natural Language Semantics 1(1). 75-116. http://dx.doi.org/10.1007/BFo2342617.

Schwarz, Berhard, Brian Buccola \& Michael Hamilton. 2012. Two types of class B numeral modifiers: A reply to Nouwen 2010. Semantics and Pragmatics 5(1). 1-25. http://dx.doi.org/10.3765/sp.5.1.

Elizabeth Coppock

Department of Philosophy, Linguistics and

Theory of Science

University of Gothenburg

Box 200

40530 Gothenburg

Sweden

eecoppock@gmail.com
Thomas Brochhagen

Abteilung für Allgemeine Sprachwissenschaft

Heinrich-Heine-Universität Düsseldorf

Universitätsstrasse 1

40225 Düsseldorf

Germany

thomas.brochhagen@hhu.de 